This item was submitted to Loughborough's Research Repository by the author.

Items in Figshare are protected by copyright, with all rights reserved, unless otherwise indicated.

\title{
Stochastic geometry analysis of large intelligent surface-assisted millimeter wave networks
}

PLEASE CITE THE PUBLISHED VERSION

https://doi.org/10.1109/JSAC.2020.3000806

PUBLISHER

IEEE

VERSION

AM (Accepted Manuscript)

\section{PUBLISHER STATEMENT}

(c) 2020 IEEE. Personal use of this material is permitted. Permission from IEEE must be obtained for all other uses, in any current or future media, including reprinting/republishing this material for advertising or promotional purposes, creating new collective works, for resale or redistribution to servers or lists, or reuse of any copyrighted component of this work in other works.

\section{LICENCE}

\section{All Rights Reserved}

\section{REPOSITORY RECORD}

Zhu, Yongxu, Gan Zheng, and Kai-Kit Wong. 2020. "Stochastic Geometry Analysis of Large Intelligent Surface-assisted Millimeter Wave Networks". Loughborough University. https://hdl.handle.net/2134/13244585.v1. 


\title{
Stochastic Geometry Analysis of Large Intelligent Surface-Assisted Millimeter Wave Networks
}

\author{
Yongxu Zhu, Member, IEEE, Gan Zheng, Senior Member, IEEE, Kai-Kit Wong, Fellow, IEEE
}

\begin{abstract}
Reliable and efficient networks are the trend for next-generation wireless communications. Recent improved hardware technologies - known as Large Intelligent Surfaces (LISs) - have decreased the energy consumption of wireless networks, while theoretically being capable of offering an unprecedented boost to the data rates and energy efficiency (EE). In this paper, we use stochastic geometry to provide performance analysis of a realistic two-step user association based millimeter wave (mmWave) networks consisting of multiple users, transmitters and one-hop reflection from a LIS. All the base stations (BSs), users and LISs are equipped with multiple uniform linear antenna arrays. The results confirm that LIS-assisted networks significantly enhance capacity and achieve higher optimal EE as compared to traditional systems when the density of BSs is not large. Moreover, there is a trade-off between the densities of LIS and BS when there is a total density constraint. It is shown that the LISs are excellent supplements for traditional cellular networks, which enormously enhance the average rate and area spectral efficiency (ASE) of mmWave networks. However, when the BS density is higher than the LIS density, the reflected interference and phase-shift energy consumption will limit the performance of LIS-assisted networks, so it is not necessary to employ the LIS devices.
\end{abstract}

Index Terms-Large intelligent surface, millimeter wave, stochastic geometry, uniform linear array.

\section{INTRODUCTION}

Mobile devices and data traffic will have grow at an unprecedented pace by 2021 [1]. To meet the high capacity requirements of future wireless communication networks, one promising solution-Large Intelligent Surface (LISs)-has attracted tremendous attention from both industry and academics. LIS is a planar array consisting of a large number of intelligent passive metallic, digital, programmable metamaterials that can obtain an improved electromagnetic propagation environment with low cost and low energy consumption [2-7]. LIS is flexible and easy to install on walls or outdoor communal facilities, that is the reason LIS is a promising solution to achieve benefit for massive multiple-input and multipleoutput (MIMO) using passive devices. When transmitters

Manuscript received September 30, 2019, revised January 16, 2020, accepted February 15, 2020. Part of this work was supported by the U.K. Engineering and Physical Sciences Research Council (EPSRC) under Grant EP/N007840/1 and EP/N008219/1. (Corresponding author: Gan Zheng)

Y. Zhu is with the Division of Computer Science and Informatics, London South Bank University, London, SE1 0AA, UK (Email: yongxu.zhu@1sbu.ac.uk).

G. Zheng is with the Wolfson School of Mechanical, Electrical and Manufacturing Engineering, Loughborough University, Leicestershire, LE11 3TU, UK (Email: g.zheng@lboro.ac.uk).

K.-K. Wong is with the Department of Electronic and Electrical Engineering, University College London, London, WC1E 6BT, UK (Email: kaikit.wong@ucl.ac.uk). communicate with receivers through intelligent metasurfaces, each element can adapt the phase shift of the electromagnetic wave to achieve the optimum performance. LIS combined with next-generation wireless communication networks creates a lot of new opportunities [2], such as reconfigurable reflector array antennae, liquid crystal metasurfaces, and hardware integration. Placing a LIS between the transmitter and receiver, and optimizing the phase shifts of elements, will maximize the signal power received, and significantly boost the performance of wireless communications [8-10].

In contrast, with the conventional antenna array concept, traditional reflecting surfaces have fixed phase shifters and it is difficult to dynamically control the signal from complicated time-varying channels [10]. Most of the current work sees LIS as an extension of MIMOs [11,12]. The work in [11] reveals that the signal through LIS after a matched-filtering (MF) beamforming may be approximated as an inter-symbol interference (ISI) channel. In [12], a LIS-based index modulation system is used to obtain high reliability along with high spectral efficiency with multiple antennae. Paper $[13,14]$ considered the ergodic rate with multiple devices and antennas in the LIS system to analyze the impact of LIS on network performance.

Energy efficiency (EE) is a particularly attractive aspect of LIS, since systems would choose passive devices instead of using directional propagation devices. [15] optimizes the base station (BS) transmit power allocation and the LIS reflector factor to maximize the EE in an LIS-based downlink multiuser multiple-input single-output (MISO) system. It is shown that the optimum result depends on the user and the number of LIS elements, and there is a trade-off between the EE and spectral efficiency (SE) in LIS-assisted communication systems. Furthermore, the traditional method of forwarding information uses a relay system. Both amplify-and-forward (AF) and decode-and-forward (DF) relaying protocols can cooperate with a BS to enhance the SE [16], and this reduces the transmit power in wireless communication networks. In addition, in current cellular infrastructures relays can be deployed more easily than small BSs, which improves efficiency and raises the coverage area [17].

It is worth pointing out that LIS technology completely differs from relay-based systems, in that a LIS does not require extra power for signal forwarding. However, each active element adapts the phase shift, and this part of energy consumption will increase with the number of elements [18]. The authors compare multiple antenna relay-assisted communication with LIS-assisted communication in [19].

Several papers propose physical layer security in LIS- 
supported systems, including [20-23], which show that the LIS technology has enormous potential to boost the security and $\mathrm{EE}$ in wireless communication networks. In [20,21,23], the authors develop the joint optimization of beamforming and phase shifts at both BS and LIS, to enhance the performance of LIS-supported physical-layer-secrecy wireless networks. [21] shows the benefit of using jamming with artificial noise (AN) to protect the secure rate of the LIS wireless system. The results in [23] show that traditional systems which don't use reflecting surfaces have limited secrecy. In [22] the authors consider a MISO system with multiple receivers and an LISsupported physical-layer-security network; the paper examines a downlink broadcast system where each eavesdropper wiretaps the data streams from both BS and the assistant LIS.

The performance of millimeter wave (mmWave) cellular networks was studied in the prior work [24], which indicates that the mmWave bands enable massive antenna arrays to be physically small for both transmitters and receivers. In [25], a stochastic geometry framework is used to model the distribution of cellular mmWave networks, which analyzes coverage and capacity in mmWave systems for outdoor infrastructure. The work in $[19,26]$ discussed the potential applications of LISs in mmWave bands. [27] solved the blockage challenge in mmWave indoor networks using reconfigurable smart reflector-arrays, and an optimal array deployment was proposed to minimize the link outage probability in mmWave communication. However, the case of LIS-supported cellular networks has not been studied.

To analyze random nodes distributed on a plane or in threedimensional cellular networks, stochastic geometry provides new perspectives on the average distribution of points in space [28]. The results from stochastic geometry bring useful insights about typical data rates and the probability of outage/coverage in cellular networks [29]. Another example that uses a stochastic geometry tool modelling point process is cell-free networks [30]. In contrast to conventional cellular networks, cell-free systems have many distributed small BSs that are jointly serving the users.

\section{A. Approach and Contributions}

The blockage effect is the main reason for reduced performance in mmWave networks [31,32], where mmWave has weak diffraction ability and low reflection coefficients [33]. In contrast, LIS-assisted mmWave networks can create a perfect line-of-sight (LoS) or holographic MIMO propagation scenario, which overcomes the blockage effect in mmWave and terahertz frequencies [2]. On the other hand, the reflecting property of LIS brings huge reflected interference which may reduce the network capacity. Therefore, it is unknown whether and under which conditions LIS in large-scale mmWave network will bring benefits or not.

In this paper, we propose a realistic LIS-assisted mmWave cellular network, where LISs coherently complement the direct links from BSs to users. To the best of our knowledge, this is the first attempt to propose a stochastic geometry framework for analyzing LIS-based mmWave cellular networks. The detailed contributions are summarized as follows.
- Two matched-filter (MF) antenna schemes. We consider a multi-antenna/element system, where all of the devices are equipped with multiple antennae or reflector elements arranged in a uniform linear array (ULA). We design two types of antenna scheme: either the antenna gain in BSs and users can be aligned by each other or aligned by the LISs. All the beamforming from the transmitters and receivers uses optimized MFs which maximizes the transmission signal. The results show that each of them has its own merits under different conditions.

- Low interference reflection model. We design a novel one-hop LIS-assisted system to avoid the signal between LISs bouncing back and forth multiple times, and we also consider the interference at each LIS which reflects the signal from all the active BSs.

- User association in the composite channel. We propose a two-step user association strategy to guarantee an efficient combination of BS, LIS and User in each slot: the first step is to determine the correct BS for a typical user, and the second step is to associate the assistant LIS with that BS. Because the composite channel needs cooperation between these two links, the approximate active densities for BSs and LISs have been considered.

- Area spectral efficiency (ASE) and EE performance analysis. We derive the analytical results of ASEs and EEs for LIS-assisted mmWave networks. For the sake of comparison, we add the traditional networks which have no support of the LISs. Our results concretely demonstrate that LIS-assisted networks achieve significant benefits over traditional networks when the density of the BSs is unable to support the requirements of the network. Moreover, optimizing the number of LIS elements achieves a maximum EE: increasing the density for BS or LIS will first degrade and then improve EE.

The rest of this paper is organized as follows. Section II presents the network model. Section III introduces the user association policy and statistical connection distances. The performance analysis and numerical result are given in Section IV and Section V, respectively. We use the following notation throughout this paper: $(\cdot)^{\mathrm{H}}$ denotes the Hermitian transpose operators. The norm of a vector $\mathbf{h}$ is denoted by $\|\mathbf{h}\|$.

\section{NETWORK MODEL}

We propose a one-hop reflective LIS-assisted large-scale mmWave cellular networks. The channels between users, BSs, and LISs are in mmWave channels that share the same frequency band. The locations of the BSs and LISs are distributed using an independent homogeneous Poisson point process (HPPP) $\Phi_{b}$ and $\Phi_{s}$ with density $\lambda_{b}$ and $\lambda_{s}$, respectively. Note that the density $\lambda_{u}$ for the locations of the users is larger than $\lambda_{b}$, and if the BS is not associated with at least one user (named inactive BS), they will switch to the idle mode. However, LISs cannot enter an idle mode because they are passive devices; these idle LISs are still active in the network as random reflective nodes, but they save the power consumption for adaptive phase shifting.

All the BSs and users are equipped with $N_{b}$ and $N_{u}$ element mmWave antennas, respectively. We assume each LIS 


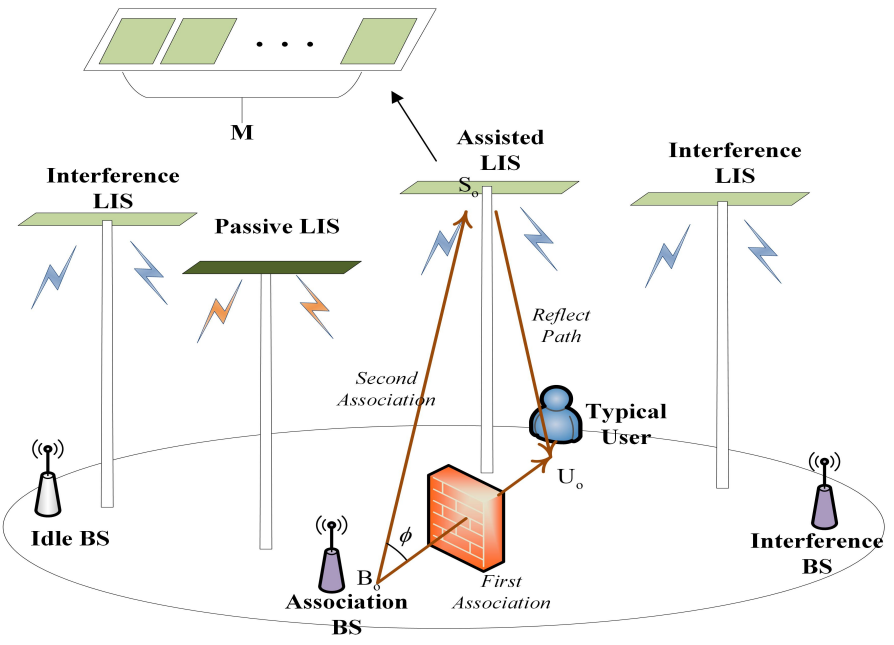

Fig. 1: Illustrative system model of the LIS-assisted mmWave networks. Each LIS equipped with $M$ elements, and the LoS link between the BSs and the users have been blocked.

is equipped with $M$ reflection antenna elements mounted on street lamps or traffic lights at the same height $H$, as illustrated in Fig. 1. The LIS element points downwards to the groundlevel devices, to avoid reflected signals among the LISs where there is only one-hop reflection from the BSs to users ${ }^{1}$.

\section{A. The Channel Model}

We derive two types of channel model, for the cases with or without LIS. Note that in the composite channel model, we assume that the reflecting surface linked to the transmitter controls the phase shifts in each time slot. Then the reflected signals can be added connectedly at the desired receiver of the user. We generally provide directional beamforming at the BSs, and users select the direct BS-User link or the BS-LISUser link, as appropriate.

1) The Direct Channel: We assume that all the nodes in the desired channel are equipped with a ULA. The steering matrix model in BS-User link is established as

$$
\mathbf{h}_{o}\left(N_{b}, \theta_{\mathrm{D}, h_{o}}, N_{u}, \theta_{\mathrm{A}, h_{o}}\right)=\mathbf{a}_{N_{u}}{ }^{\mathrm{H}}\left(\theta_{\mathrm{A}, h_{o}}\right) \mathbf{a}_{N_{b}}\left(\theta_{\mathrm{D}, h_{o}}\right) \text {, }
$$

with

$$
\left\{\begin{array}{l}
\mathbf{a}_{N_{b}}\left(\theta_{\mathrm{D}, h_{o}}\right)=\left[1, e^{j 2 \pi \frac{\Delta d}{\omega} \sin \theta_{\mathrm{D}, h_{o}}}, . ., e^{j 2 \pi \frac{\Delta d}{\omega}\left(N_{b}-1\right) \sin \theta_{\mathrm{D}, h_{o}}}\right] \\
\mathbf{a}_{N_{u}}\left(\theta_{\mathrm{A}, h_{o}}\right)=\left[1, e^{j 2 \pi \frac{\Delta d}{\omega} \sin \theta_{\mathrm{A}, h_{o}}}, . ., e^{j 2 \pi \frac{\Delta d}{\omega}\left(N_{u}-1\right) \sin \theta_{\mathrm{A}, h_{o}}}\right],
\end{array}\right.
$$

where all the antenna elements are placed along the propagation plane with $\Delta d=\omega / 2$ spacing, where $\omega$ is the wavelength. $\theta_{\mathrm{D}, h_{o}} \sim U(0,2 \pi)$ and $\theta_{\mathrm{A}, h_{o}} \sim U(0,2 \pi)$ are the azimuth angle of departure (AoD) and the angle of arrival (AoA), respectively. We model the MF optimal analog beamforming

\footnotetext{
${ }^{1}$ We assume the parallel structure for all the intelligent surfaces, which can effectively avoid the reflected interference between multiple LISs.
}

which is adopted at the BSs and users for maximizing the received signal power in the desired link and as

$$
\left\{\begin{array}{l}
\mathbf{w}_{N_{b}}\left(\theta_{\mathrm{D}}\right)=\sqrt{\frac{1}{N_{b}}} \mathbf{a}_{N_{b}}\left(\theta_{\mathrm{D}}\right) \\
\mathbf{w}_{N_{u}}\left(\theta_{\mathrm{A}}\right)=\sqrt{\frac{1}{N_{u}}} \mathbf{a}_{N_{u}}\left(\theta_{\mathrm{A}}\right)
\end{array} .\right.
$$

We define two types of antenna schemes as follows.

- The first antenna scheme aims to optimize the direct channel, where the MF angle seen by the serving BS $\theta_{\mathrm{A}}$ and typical user $\theta_{\mathrm{D}}$ will be equal to the BS-User channel angles $\theta_{\mathrm{A}, h_{o}}$ and $\theta_{\mathrm{D}, h_{o}}$. Then we have the optimized antenna gain $\mathcal{N}_{(1)}$ for a typical user seen by its serving BS as

$$
\begin{aligned}
& \mathcal{N}_{(1)}= \\
& \left|\mathbf{w}_{N_{u}}\left(\theta_{\mathrm{A}, h_{o}}\right) \mathbf{h}_{o}\left(N_{b}, \theta_{\mathrm{D}, h_{o}}, N_{u}, \theta_{\mathrm{A}, h_{o}}\right) \mathbf{w}_{N_{b}}{ }^{\mathrm{H}}\left(\theta_{\mathrm{D}, h_{o}}\right)\right|^{2} \\
& =N_{b} N_{u} .
\end{aligned}
$$

- The second antenna scheme aims to optimize the LIS channel, where the MF angle seen by the serving BS $\theta_{\mathrm{A}}$ and typical user $\theta_{\mathrm{D}}$ will be equal to the BS-LIS channel angle $\theta_{\mathrm{A}, g_{o}}$ and the LIS-User channel angle $\theta_{\mathrm{D}, k_{o}}$, respectively. The array gain as follows

$$
\begin{aligned}
& \mathcal{N}_{(2)}= \\
& \left|\mathbf{w}_{N_{u}}\left(\theta_{\mathrm{A}, g_{o}}\right) \mathbf{h}_{o}\left(N_{b}, \theta_{\mathrm{D}, h_{o}}, N_{u}, \theta_{\mathrm{A}, h_{o}}\right) \mathbf{w}_{N_{b}}{ }^{\mathrm{H}}\left(\theta_{\mathrm{D}, k_{o}}\right)\right|^{2} .
\end{aligned}
$$

After using Euler's formula and trigonometric identities, $\mathcal{N}_{(2)}$ can be expressed as

$$
\begin{aligned}
& \mathcal{N}_{(2)}=\frac{1}{N_{b} N_{u}} \times \\
& \frac{1-\cos \left(N_{b} \mathcal{G}\left(\theta_{\mathrm{D}, h_{o}}, \theta_{\mathrm{D}, k_{o}}\right)\right)}{1-\cos \left(\mathcal{G}\left(\theta_{\mathrm{D}, h_{o}}, \theta_{\mathrm{D}, k_{o}}\right)\right)} \frac{1-\cos \left(N_{u} \mathcal{G}\left(\theta_{\mathrm{A}, h_{o}}, \theta_{\mathrm{A}, g_{o}}\right)\right)}{1-\cos \left(\mathcal{G}\left(\theta_{\mathrm{A}, h_{o}}, \theta_{\mathrm{A}, g_{o}}\right)\right)}
\end{aligned}
$$

where we have $\mathcal{G}\left(\theta_{1}, \theta_{2}\right)=2 \pi \frac{\Delta d}{\omega}\left(\sin \theta_{1}-\sin \theta_{2}\right)$. The antenna gain from an active interference BS $B_{i}$ seen by the typical user can be obtained as

$$
\begin{aligned}
& \mathcal{N}_{i}=\left|\mathbf{w}_{N_{u}}\left(\theta_{\mathrm{A}, h_{o} / g_{o}}\right) \mathbf{h}_{i}\left(N_{b}, \theta_{\mathrm{D}, h_{i}}, N_{u}, \theta_{\mathrm{A}, h_{i}}\right) \mathbf{w}_{N_{b}}{ }^{\mathrm{H}}\left(\theta_{\mathrm{D}}\right)\right|^{2} \\
& =\underbrace{\left|\mathbf{w}_{N_{u}}\left(\theta_{\mathrm{A}, h_{o} / g_{o}}\right) \mathbf{a}_{N_{u}}{ }^{\mathrm{H}}\left(\theta_{\mathrm{A}, h_{i}}\right)\right|^{2}}_{\bar{n}_{u}} \underbrace{\left|\mathbf{a}_{N_{b}}\left(\theta_{\mathrm{D}, h_{i}}\right) \mathbf{w}_{N_{b}}{ }^{\mathrm{H}}\left(\theta_{\mathrm{D}}\right)\right|^{2}}_{\bar{n}_{b}},
\end{aligned}
$$

where $\mathbf{h}_{i}$ denotes the steering matrix from the interfering BS $\mathrm{B}_{i}$ to the user link. For convenience, we decompose $\mathcal{N}_{i}=$ $\bar{n}_{b} \bar{n}_{u}$, where $\bar{n}_{u}$ is the average interference antenna gain of typical user, which can be expressed as

$$
\begin{aligned}
& \bar{n}_{u}=\mathbb{E}\left[\frac{1-\cos \left(N_{u} \mathcal{G}\left(\theta_{\mathrm{A}, h_{i}}, \theta_{\mathrm{A}, h_{o} / g_{o}}\right)\right)}{1-\cos \left(\mathcal{G}\left(\theta_{\mathrm{A}, h_{i}}, \theta_{\mathrm{A}, h_{o} / g_{o}}\right)\right)}\right] \\
& =\frac{1}{N_{u}} \frac{1}{2 \pi} \int_{0}^{2 \pi} \frac{1-\cos \left(N_{u} \mathcal{G}\left(\theta_{\mathrm{A}, h_{i}}, \theta_{\mathrm{A}, h_{o} / g_{o}}\right)\right)}{1-\cos \left(\mathcal{G}\left(\theta_{\mathrm{A}, h_{i}}, \theta_{\mathrm{A}, h_{o} / g_{o}}\right)\right)} d \theta_{\mathrm{A}, h_{i}}
\end{aligned}
$$


where the AoA in typical user has got to be either $\theta_{\mathrm{A}, h_{o}}$ or $\theta_{\mathrm{A}, g_{o}} \cdot \bar{n}_{b}$ is the average interference antenna gain of the interfering $\mathrm{BS}$ given as

$$
\begin{aligned}
\bar{n}_{b} & =\mathbb{E}\left[\frac{1-\cos \left(N_{b} \mathcal{G}\left(\theta_{\mathrm{D}, h_{i}}, \theta_{\mathrm{D}, h_{o}}\right)\right)}{1-\cos \left(\mathcal{G}\left(\theta_{\mathrm{D}, h_{i}}, \theta_{\mathrm{D}, h_{o}}\right)\right)}\right]=\frac{1}{N_{b}} \frac{1}{4 \pi^{2}} \\
& \times \int_{0}^{2 \pi} \int_{0}^{2 \pi} \frac{1-\cos \left(N_{b} \mathcal{G}\left(\theta_{\mathrm{D}, h_{i}}, \theta_{\mathrm{A}, h_{o}}\right)\right)}{1-\cos \left(\mathcal{G}\left(\theta_{\mathrm{D}, h_{i}}, \theta_{\mathrm{D}, h_{o}}\right)\right)} d \theta_{\mathrm{D}, h_{i}} d \theta_{\mathrm{D}, h_{o}} .
\end{aligned}
$$

2) The LIS assisted channel: We derive the ULA steering matrix model in the LIS-assisted channel at the BS-to-LIS link and LIS-to-User link respectively, which are expressed as

$$
\left\{\begin{array}{l}
\mathbf{k}_{o}\left(N_{b}, \theta_{\mathrm{D}, k_{o}}, M, \theta_{\mathrm{A}, k_{o}}\right)=\mathbf{a}_{M}{ }^{\mathrm{H}}\left(\theta_{\mathrm{A}, k_{o}}\right) \mathbf{a}_{N_{b}}\left(\theta_{\mathrm{D}, k_{o}}\right) \\
\mathbf{g}_{o}\left(M, \theta_{\mathrm{D}, g_{o}}, N_{u}, \theta_{\mathrm{A}, g_{o}}\right)=\mathbf{a}_{N_{u}}{ }^{\mathrm{H}}\left(\theta_{\mathrm{A}, g_{o}}\right) \mathbf{a}_{M}\left(\theta_{\mathrm{D}, g_{o}}\right)
\end{array}\right.
$$

with

$$
\mathbf{a}_{M}(\vartheta)=\left[1, e^{j 2 \pi \frac{\Delta d}{\lambda} \sin \vartheta}, . ., e^{j 2 \pi \frac{\Delta d}{\lambda}(M-1) \vartheta}\right]
$$

where $\vartheta=\left\{\theta_{\mathrm{A}, k_{o}}, \theta_{\mathrm{D}, g_{o}}\right\}$ are the AoA in the BS-to-LIS channel or AoD in the LIS-to-user channel, and $\theta_{\mathrm{A}, k}^{b}$ is the AoA in the LIS channel adopted at the BS, and $\theta_{\mathrm{D}, g}^{u}$ is the AoD in the LIS channel adopted at the user. When the signal is reflected by the specified LIS, the phases of the signals in this LIS can be adapted as needed. The steering matrix model in the BS-to-user link via a LIS is given by

$$
\begin{aligned}
\widehat{\mathbf{h}}_{o} & \left(N_{b}, \theta_{\mathrm{D}, k_{o}}, N_{u}, \theta_{\mathrm{A}, g_{o}}\right)=\mathbf{g}_{o} \boldsymbol{\Theta} \mathbf{k}_{o} \\
& =\mathbf{a}_{N_{u}}{ }^{\mathrm{H}}\left(\theta_{\mathrm{A}, g_{o}}\right) \mathbf{a}_{M}\left(\theta_{\mathrm{D}, g_{o}}\right) \boldsymbol{\Theta} \mathbf{a}_{M}{ }^{\mathrm{H}}\left(\theta_{\mathrm{A}, k_{o}}\right) \mathbf{a}_{N_{b}}\left(\theta_{\mathrm{D}, k_{o}}\right)
\end{aligned}
$$

where $\Theta$ is the phase shift diagonal matrix introduced by the $m$-th element of the LIS as

$$
\boldsymbol{\Theta}=\operatorname{diag}\left(\beta e^{j \varphi_{1}}, \ldots, \beta e^{j \varphi_{m}}, \ldots, \beta e^{j \varphi_{M}}\right),
$$

where $\varphi_{m} \in[0,2 \pi)$ denotes the phase shift introduced by the $m$-th element of the LIS. $\beta \in(0,1]$ denotes the reflection efficiency, and we set as $\beta=1[9,34]$.

We define $\Theta_{o}$ as the beamforming weight introduced by the LIS, which aims to maximize the transmitting signal in the assisted channel to optimize the phase shift design. Since that the ergodic spectral efficiency depends on $\mathbf{a}_{M}\left(\theta_{\mathrm{D}, g_{o}}\right)$ and $\mathbf{a}_{M}\left(\theta_{\mathrm{A}, k_{o}}\right)$ only [9], we obtain the optimal $\boldsymbol{\Theta}_{o}$ with $\varphi_{m, o}^{*}$ satisfies

$$
\max _{\varphi_{m, o}} \delta_{L}=\mathbf{a}_{M}\left(\theta_{\mathrm{D}, g_{o}}\right) \boldsymbol{\Theta}_{\mathbf{o}} \mathbf{a}_{M}{ }^{\mathrm{H}}\left(\theta_{\mathrm{A}, k_{o}}\right),
$$

then, we can derive that

$$
\delta_{L}=\left|\begin{array}{ccc}
\varphi_{1} & \cdots & 0 \\
\vdots & \ddots & \\
0 & & \varphi_{M} e^{j 2 \pi \frac{\Delta d}{\omega}(m-1)\left(\sin \theta_{\mathrm{D}, g_{o}}-\sin \theta_{\mathrm{A}, k_{o}}\right)}
\end{array}\right|,
$$

In order to achieve the maximal $\delta_{L}$, the optimal phase shift on the $m$-th antenna element of the assisted LIS can be given by

$$
\varphi_{m, o}^{*}=2 \pi \frac{\Delta d}{\omega}(m-1)\left(\sin \theta_{\mathrm{D}, g_{o}}-\sin \theta_{\mathrm{A}, k_{o}}\right)
$$

Compared to the direct link, we consider the two types of antenna schemes below.

- The adapted second antenna scheme aims to optimize the LIS channel, the MF angle in the BS and typical user towards the reflected channel like (4). The antenna gain via the assisted LIS is as follows

$$
\begin{aligned}
\widehat{\mathcal{N}}_{(2)} & =\left|\mathbf{w}_{N_{u}}\left(\theta_{\mathrm{A}, g_{o}}\right) \mathbf{g}_{o} \boldsymbol{\Theta}_{\mathbf{o}} \mathbf{k}_{o} \mathbf{w}_{N_{b}}{ }^{\mathrm{H}}\left(\theta_{\mathrm{D}, k_{o}}\right)\right|^{2} \\
& =M^{2} N_{b} N_{u} .
\end{aligned}
$$

- The corresponding first antenna scheme in the LISassisted channel denotes the MF in the BS and user towards the desired channel like (3), and the antenna scheme via the assisting LIS is

$$
\begin{aligned}
\widehat{\mathcal{N}}_{(1)} & =\left|\mathbf{w}_{N_{u}}\left(\theta_{\mathrm{A}, h_{o}}\right) \mathbf{g}_{o} \boldsymbol{\Theta}_{\mathbf{o}} \mathbf{k}_{o} \mathbf{w}_{N_{b}}{ }^{\mathrm{H}}\left(\theta_{\mathrm{D}, h_{o}}\right)\right|^{2} \\
& =M^{2} \bar{n}_{b} \bar{n}_{u} .
\end{aligned}
$$

For any one BS-to-user link, via the interfering LISs the average array gain is given by

$$
\begin{aligned}
& \widehat{\mathcal{N}}_{q / i}=|\mathbf{w}_{N_{u}}\left(\theta_{\mathrm{A}, h_{o} / g_{o}}\right) \underbrace{\mathbf{g}_{i} \boldsymbol{\Theta}_{q / i} \mathbf{k}_{i}}_{\widehat{\mathbf{h}}_{i}} \mathbf{w}_{N_{b}} \mathrm{H}\left(\theta_{\mathrm{D}}\right)|^{2} \\
& =\underbrace{\left|\mathbf{w}_{N_{u}}\left(\theta_{\mathrm{A}, h_{o} / g_{o}}\right) \mathbf{a}_{N_{u}} \mathrm{H}\left(\theta_{\mathrm{A}, g_{i}}\right)\right|^{2}}_{\bar{n}_{u}} \\
& \times \underbrace{\left|\mathbf{a}_{M}\left(\theta_{\mathrm{D}, g_{i}}\right) \boldsymbol{\Theta}_{q / i} \mathbf{a}_{M} \mathrm{H}\left(\theta_{\mathrm{A}, k_{i}}\right)\right|^{2}}_{\chi_{q / i}} \underbrace{\left|\mathbf{a}_{N_{b}}\left(\theta_{\mathrm{D}, k_{i}}\right) \mathbf{w}_{N_{b}}^{\mathrm{H}}\left(\theta_{\mathrm{D}}\right)\right|^{2}}_{\bar{n}_{b}},
\end{aligned}
$$

where $\widehat{\mathbf{h}}_{i}=\mathbf{g}_{i} \boldsymbol{\Theta}_{q / i} \mathbf{k}_{i}$ denotes the steering matrix model from the interfering BS-to-user links via interfering LISs, $\widehat{\mathcal{N}}_{q}$ and $\widehat{\mathcal{N}}_{i}$ indicate the antenna gain from idle interfering LISs and active LISs which are associated with other users, respectively. $\bar{n}_{b}$ and $\bar{n}_{u}$ are the average interference antenna gains for the BSs and users, respectively. We consider two types of phase shift diagonal matrix: $\boldsymbol{\Theta}_{q}$ with $\varphi_{m, q}$, and $\boldsymbol{\Theta}_{i}$ with $\varphi_{m, i}$ indicate the random array reflection gain from idle LISs and optimized array reflection gain from active LISs, respectively. Due to the fact that LISs are passive devices, they participate in the reflection process with random phase shift $\varphi_{m, q} \in[0,2 \pi]$, even those not selected to support the BS and user links. We define $\chi_{q}$ as the random antenna reflection gain from idle LISs, which can be expressed as

$$
\begin{aligned}
& \chi_{q}=\left|\mathbf{a}_{M}\left(\theta_{\mathrm{D}, g_{i}}\right) \boldsymbol{\Theta}_{q} \mathbf{a}_{M}{ }^{\mathrm{H}}\left(\theta_{\mathrm{A}, k_{i}}\right)\right|^{2} \\
& =\mathbb{E}\left[\left|\sum_{m=1}^{M} \beta e^{j\left(2 \pi \frac{\Delta d}{\omega}(m-1)\left[\sin \theta_{\mathrm{D}, g_{i}}-\sin \theta_{\mathrm{A}, k_{i}}\right]+\varphi_{m, q}\right)}\right|^{2}\right] .
\end{aligned}
$$

Note that $\chi_{i}$ is the optimized antenna reflection gain from other active LISs, and these LISs are serving their own BSUser $i$-th pair, and the random phase shift follows as

$$
\varphi_{m, i}^{*}=2 \pi \frac{\Delta d}{\omega}(m-1)\left(\sin \theta_{\mathrm{D}, g_{i, o}}-\sin \theta_{\mathrm{A}, k_{i, o}}\right),
$$


where $\theta_{\mathrm{D}, g_{i, o}}$ and $\theta_{\mathrm{A}, k_{i, o}}$ are the optimized phase shift in the channel $\mathbf{g}_{i}$ and $\mathbf{k}_{i}$, respectively. Then we derive $\chi_{i}$ as

$$
\begin{aligned}
& \chi_{i}=\left|\mathbf{a}_{M}\left(\theta_{\mathrm{D}, g_{i}}\right) \boldsymbol{\Theta}_{i} \mathbf{a}_{M}{ }^{\mathrm{H}}\left(\theta_{\mathrm{A}, k_{i}}\right)\right|^{2} \\
& =\sum_{m=1}^{M} \beta e^{j(m-1)\left[\mathcal{G}\left(\theta_{\mathrm{D}, g_{i}}, \theta_{\mathrm{D}, g_{i, o}}\right)-\mathcal{G}\left(\theta_{\mathrm{A}, k_{i, o}}, \theta_{\mathrm{A}, k_{i}}\right)\right]} \\
& =\int_{0}^{2 \pi} \int_{0}^{2 \pi} \int_{0}^{2 \pi} \int_{0}^{2 \pi} \frac{\beta}{16 \pi^{4}} \\
& \times \hat{\mathcal{G}}\left(\theta_{\mathrm{D}}^{s}, \theta_{\mathrm{A}}^{s}, \theta_{\mathrm{D}, \mathrm{i}}^{s}, \theta_{\mathrm{A}, \mathrm{i}}^{s}\right) d \theta_{\mathrm{D}}^{s} d \theta_{\mathrm{A}}^{s} d \theta_{\mathrm{D}, \mathrm{i}}^{s} d \theta_{\mathrm{A}, \mathrm{i}}^{s} .
\end{aligned}
$$

with

$$
\begin{aligned}
\hat{\mathcal{G}}\left(\theta_{\mathrm{D}}^{s}, \theta_{\mathrm{A}}^{s}, \theta_{\mathrm{D}, \mathrm{i}}^{s}, \theta_{\mathrm{A}, \mathrm{i}}^{s}\right) \\
=\frac{1-\cos \left(M\left[\mathcal{G}\left(\theta_{\mathrm{D}, g_{i}}, \theta_{\mathrm{D}, g_{i, o}}\right)-\mathcal{G}\left(\theta_{\mathrm{A}, k_{i, o}}, \theta_{\mathrm{A}, k_{i}}\right)\right]\right)}{1-\cos \left(\mathcal{G}\left(\theta_{\mathrm{D}, g_{i}}, \theta_{\mathrm{D}, g_{i, o}}\right)-\mathcal{G}\left(\theta_{\mathrm{A}, k_{i, o}}, \theta_{\mathrm{A}, k_{i}}\right)\right)} .
\end{aligned}
$$

\section{B. Downlink Transmission}

We assume that perfect channel state information (CSI) is known at the BSs in the mmWave-based LIS system. The blockage effects in the LIS signal path can be LoS or Nonline-of-sight (NLoS). We denote $p_{\mathrm{L}}(r)$ as the probability that a link at a distance $r$ is LoS, while the NLoS probability of a link is $1-p_{\mathrm{L}}(r)$. We assume a very high blockage effect in the ground networks [35] and the probability of LoS link approaches 0 between users and BSs on the ground. Based on the above reasoning, we approximate that all links on the ground are NLoS. For tractability, we neglect smallscale fading because it is not significant in LoS links in mmWave [31]. Since mmWave channels have spatial sparsity in the angle domain, the multipath components in mmWave are mainly from reflections rather than refraction or scattering [36-38].

For the received signal power of the direct link, it can be evaluated as

$$
\mathcal{S}_{\mathrm{S}, d}=P_{T}\left|\mathbf{h}_{o}\right|^{2} L\left(x_{o}\right)=P_{T} \mathcal{N}_{1} L\left(x_{o}\right),
$$

where $L\left(x_{o}\right)$ is path loss fading function with $L\left(x_{o}\right)=$ $C x_{o}{ }^{-\alpha_{\mathrm{B}}}, x_{o}$ is the distance from the typical user to the serving BS, $C$ is the path-loss intercept factor, which is commonly set as $20 \log _{10}\left(\frac{2 \pi f_{c}}{c}\right), f_{c}$ is the carrier frequency, and $c=3 \times 10^{8} \mathrm{~m} / \mathrm{s}$ [32]. $P_{T}$ denotes the transmit power from the BS. Now consider the signal reflected by the LIS: the received signal power to the typical user can be computed as

$$
\mathcal{S}_{\mathrm{S}, o}=P_{T}\left|\mathbf{w}_{N_{u}}\left(\theta_{\mathrm{A}}\right) \widetilde{\mathbf{h}}_{o} \mathbf{w}_{N_{b}}{ }^{\mathrm{H}}\left(\theta_{\mathrm{D}}\right)\right|^{2}
$$

where $\widetilde{\mathbf{h}}_{o}$ is the composite channel model as follows

$$
\widetilde{\mathbf{h}}_{o}=\widehat{\mathbf{h}}_{o} \sqrt{L\left(y_{o}\right)} \sqrt{L\left(z_{o}\right)}+\mathbf{h}_{o} \sqrt{L\left(x_{o}\right)} .
$$

Then we derive the received signal with antenna scheme 1, which is given by

$$
\begin{aligned}
\mathcal{S}_{\mathrm{S}, 1} & =P_{T} C\left|\mathbf{w}_{N_{u}}\left(\theta_{\mathrm{A}, h_{o}}\right) \widetilde{\mathbf{h}}_{o} \mathbf{w}_{N_{b}}{ }^{\mathrm{H}}\left(\theta_{\mathrm{D}, h_{o}}\right)\right|^{2} \\
& =P_{T}\left(\sqrt{\widehat{\mathcal{N}}_{(1)} L\left(y_{o}\right) L\left(z_{o}\right)}+\sqrt{\mathcal{N}_{(1)} L\left(x_{o}\right)}\right)^{2} .
\end{aligned}
$$

Similarly, we derive the received signal with antenna scheme 2 , which is given by

$$
\begin{aligned}
\mathcal{S}_{\mathrm{S}, 2} & =P_{T}\left|\mathbf{w}_{N_{u}}\left(\theta_{\mathrm{A}, g_{o}}\right) \widetilde{\mathbf{h}}_{o} \mathbf{w}_{N_{b}}{ }^{\mathrm{H}}\left(\theta_{\mathrm{D}, k_{o}}\right)\right|^{2} \\
& =P_{T}\left(\sqrt{\widehat{\mathcal{N}}_{(2)} L\left(y_{o}\right) L\left(z_{o}\right)}+\sqrt{\mathcal{N}_{(2)} L\left(x_{o}\right)}\right)^{2}
\end{aligned}
$$

where $L\left(r_{o}\right)$ is the path loss fading function with $L\left(r_{o}\right)=$ $C r_{o}^{-\alpha_{v}}$, and $r_{o}$ denotes the distance. $y_{o}$ is the distance from the assisting LIS to the typical user and $z_{O}$ is the distance from the associated BS to the assisting LIS. $\alpha_{v}$ is the path loss exponent depending on the LoS or NLoS link, namely $\alpha_{v}=\alpha_{\mathrm{L}}$ for the LoS link and $\alpha_{v}=\alpha_{\mathrm{NL}}$ for the NLoS link.

Hence, the direct and LIS-assisted signal-to-interferenceplus-noise ratio (SINR) at a typical receiver is given as

$$
\left\{\begin{array}{l}
\gamma_{\mathrm{S}, d}=\mathcal{S}_{\mathrm{S}, d} /\left(\mathcal{I}_{\mathrm{B}}+\mathcal{I}_{\mathrm{S}}+\sigma^{2}\right), \\
\gamma_{\mathrm{S}, o}=\mathcal{S}_{\mathrm{S}, o} /\left(\mathcal{I}_{\mathrm{B}}+\mathcal{I}_{\mathrm{S}}+\sigma^{2}\right),
\end{array}\right.
$$

where $\mathcal{I}_{B}$ is the interference from all the active BSs and can be evaluated as

$$
\mathcal{I}_{\mathrm{B}}=\sum_{i \in \Phi_{b}^{A c t} \backslash o}\left\|\mathbf{h}_{i}\right\|^{2} L\left(x_{i}\right)
$$

where $x_{i}$ is the distance between the typical receiver and the active interferer BSs set $\mathrm{B}_{i} \in \Phi_{b}^{A c t}$. $\mathcal{I}_{S}$ is the interference from all the LISs in the system, given by

$$
\mathcal{I}_{\mathrm{S}}=\sum_{i \in \Phi_{\mathrm{s}}}\left|\Xi_{i} \boldsymbol{\Theta}_{q \backslash i} \mathbf{g}_{i}\right|^{2} L\left(y_{i}\right)
$$

where $\Xi_{i}=\sum_{j \in \Phi_{\mathrm{b}}}\left|\mathbf{k}_{i_{j}}\right|^{2} L\left(z_{i_{j}}\right)$ is the interference from active BSs which is reflected by the interfering LISs $\mathrm{S}_{i}$, and then $\mathrm{S}_{i}$ reflects the summarized interference to the typical user. $y_{i}$ is the distance from the $\mathrm{S}_{i}$ to the typical user, $z_{i_{j}}$ is the distance from the interfering $\mathrm{BS} \mathrm{B}_{j}$ to $\mathrm{S}_{i} . \sigma^{2}$ is the noise power.

\section{User Association Policy and Statistical CONnECTION DistancES}

In this section, we analyze the two-step association probability in the composite channel networks. Given the typical user association with one BS for signal receiving, we consider this as the first step in user association. For the second step in user association, the aim of the LIS is to minimize the uplink path loss from the serving BS to the assisted LIS, in order to strengthen the assisted channel.

\section{A. First Association}

In the first step, a typical user $\mathrm{U}_{O}$ is typically associated with the nearest $\mathrm{BS}$, named $\mathrm{B}_{o}$. The ground $\mathrm{BS}$ density and transmit power determine the association probability for a typical user. The probability density function (PDF) of the distance $x_{o}$ between a typical user and its nearest serving BS can be written as [39]

$$
f_{\mathrm{B}}\left(x_{o}\right)=2 \pi \lambda_{b} x_{o} e^{-\pi \lambda_{b} x_{o}^{2}} .
$$

In practice, if the density of users is not much higher than that of the BSs, there may exist the possibility of empty cell probability, which corresponds to the case that no user is 
associated to this $\mathrm{BS}$ at this moment. We mathematically use $p_{v 1}$ to present the empty-cell probability, which can be written as

$p_{v 1}\left(\lambda_{b}, \lambda_{u}\right)=\int_{0}^{\infty} e^{-\lambda_{u} x} f_{\mathcal{S}, b}\left(x, \lambda_{b}\right) d x \approx\left(1+\frac{\lambda_{u}}{3.5 \lambda_{b}}\right)^{-3.5}$,

where $f_{\mathcal{S}, b}\left(x, \lambda_{b}\right)$ is the approximated distribution function of an area $\mathcal{S}$, and the area of a typical Voronoi cell in a Poisson random tessellation [40] is expressed as

$$
f_{\mathcal{S}, b}\left(x, \lambda_{b}\right) \approx \frac{3.5^{3.5}}{\Gamma(3.5)} \lambda_{b}{ }^{3.5} x^{2.5} e^{-3.5 \lambda_{b} x} .
$$

Corollary 1: We define the density $\lambda_{b}^{1}=p_{v 1} \lambda_{b}$ as the thinning process in $\Phi_{b}$ for the idle BSs as shown in Fig. 2. It indicates that the idle BSs are not the closest BS for any user in this system ${ }^{2} \cdot \lambda_{b}^{0}=\left(1-p_{v 1}\right) \lambda_{b}$ is the density of active BS-User pairs. Note that the empty cell probability $p_{v 1}$ will approach to 0 since $\lambda_{u} \gg \lambda_{b}$.

\section{B. Second Association}

In the second step in association, the typical user $\mathrm{U}_{o}$ is associated with the nearest LIS with the minimum path loss, named $\mathrm{S}_{o}$. We will equivalently consider $\Phi_{s}^{\mathrm{L}}+\Phi_{s}^{\mathrm{NL}}=\Phi_{s}$ as two independent sets of LISs, where the assisting LIS can be either the smallest path loss fading LIS with higher LoS link in $\Phi_{s}^{\mathrm{L}}$ or the smallest path loss fading LIS with higher NLoS link in $\Phi_{s}^{\mathrm{NL}}$.

We assume different path loss laws are applied to LoS or NLoS links. Based on the given distance $y_{o}$, the path loss gain is as follows

$$
L\left(y_{o}\right)=p_{\mathrm{L}}\left(\widehat{y}_{o}\right) y_{o}^{\alpha_{\mathrm{L}}}+p_{\mathrm{NL}}\left(\widehat{y}_{o}\right) y_{o}^{\alpha_{\mathrm{NL}}},
$$

where $\alpha_{\mathrm{L}}$ and $\alpha_{\mathrm{NL}}$ are the LoS and NLoS path loss exponents, respectively, and $\widehat{y}_{o}=\sqrt{y_{o}^{2}-H^{2}}$ is the projection distance from the serving BS to the associated LIS's projecting position, $p_{\mathrm{L}}\left(\widehat{y}_{o}\right)$. The LoS connection probability [41] is as follows

$$
\left\{\begin{array}{l}
p_{\mathrm{L}}\left(\widehat{y}_{o}\right)=\frac{1}{1+a \exp \left(-b\left[\arctan \left(\frac{H}{\widehat{y}_{o}}\right)-a\right]\right)} . \\
p_{\mathrm{NL}}\left(\widehat{y}_{o}\right)=1-p_{\mathrm{L}}\left(\widehat{y}_{o}\right)
\end{array}\right.
$$

Based on the above policy, we find the following lemmas useful.

Lemma 1: Under the given distance $y_{o}, f_{\mathrm{S}}^{(\mathrm{L})}\left(y_{o}\right)$ is the PDF of the smallest path loss fading assisting LIS's distance with LoS link among the typical user, which can be expressed as follows

$$
\begin{aligned}
& f_{\mathrm{S}}^{(\mathrm{L})}\left(y_{o}\right)=\frac{2 \pi \lambda_{s} y}{\Lambda_{\mathrm{S}}^{(\mathrm{L})}} p_{\mathrm{L}}\left(\sqrt{y^{2}-H^{2}}\right) \\
& \times e^{-2 \pi \lambda_{s}\left(\int_{H}^{\frac{\alpha_{\mathrm{L}}}{\alpha_{\mathrm{N}}}} t p_{\mathrm{NL}}\left(\sqrt{t^{2}-H^{2}}\right) d t+\int_{H}^{y} t p_{\mathrm{L}}\left(\sqrt{t^{2}-H^{2}}\right) d t\right)},
\end{aligned}
$$

\footnotetext{
${ }^{2}$ For the convenience of calculation, we assume that unconnected users with density $\lambda_{u}^{1}=\lambda_{u}-\left(1-p_{v 1}\right) \lambda_{b}$ will be in the idle mode and waiting for the service in the next time slot.
}

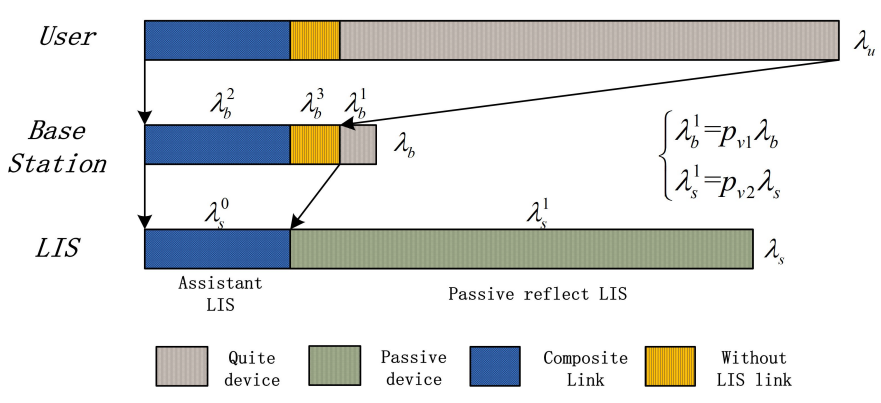

Fig. 2: Empty cell density diagram.

where $\Lambda_{\mathrm{S}}^{(\mathrm{L})}$ is the LoS connection probability that a typical user is associated with the LIS, we obtain

$$
\begin{aligned}
\Lambda_{\mathrm{S}}^{(\mathrm{L})} & =2 \pi \lambda_{s} \int_{H}^{\infty} y p_{\mathrm{L}}\left(\sqrt{y^{2}-H^{2}}\right) \\
& \times e^{-2 \pi \lambda_{\mathrm{L}}\left(\int_{H}^{\frac{\alpha_{\mathrm{L}}}{\alpha_{N}}} t p_{\mathrm{NL}}\left(\sqrt{t^{2}-H^{2}}\right) d t+\int_{H}^{y} t p_{\mathrm{L}}\left(\sqrt{t^{2}-H^{2}}\right) d t\right)} d y .
\end{aligned}
$$

Lemma 2: Under the given distance $y_{o}$, we have the NLoS PDF of the minimum path loss between the typical user and the assisting LIS given by

$$
\begin{aligned}
& f_{\mathrm{S}}^{(\mathrm{NL})}\left(y_{o}\right)=\frac{2 \pi \lambda_{s} y}{\Lambda_{\mathrm{S}}^{(\mathrm{NL})}} p_{\mathrm{NL}}\left(\sqrt{y^{2}-H^{2}}\right) \\
& \times e^{-2 \pi \lambda_{s}\left(\int_{H}^{y} t p_{\mathrm{NL}}\left(\sqrt{t^{2}-H^{2}}\right) d t+\int_{H}^{\frac{\alpha_{\mathrm{N}}}{\alpha \mathrm{L}}} t p_{\mathrm{L}}\left(\sqrt{t^{2}-H^{2}}\right) d t\right)},
\end{aligned}
$$

where $\Lambda_{\mathrm{S}}^{(\mathrm{NL})}$ is the LoS connection probability that a typical user is associated with the LIS, given as

$$
\begin{aligned}
\Lambda_{\mathrm{S}}^{(\mathrm{NL})} & =2 \pi \lambda_{s} \int_{H}^{\infty} y p_{\mathrm{NL}}\left(\sqrt{y^{2}-H^{2}}\right) \\
& \times e^{-2 \pi \lambda_{s}\left(\int_{H}^{y} t p_{\mathrm{NL}}\left(\sqrt{t^{2}-H^{2}}\right) d t+\int_{H}^{\frac{\alpha_{\mathrm{N}}}{\alpha_{\mathrm{L}}}} t p_{\mathrm{L}}\left(\sqrt{t^{2}-H^{2}}\right) d t\right.} d y .
\end{aligned}
$$

Lemma 3: For the second association, the empty cell probability of the LIS process for a typical Voronoi cell can be approximated as

$$
p_{v 2} \stackrel{(b)}{\approx} \int_{0}^{\infty} e^{-\lambda_{b}^{0} x} f_{\mathcal{S}}\left(x, \lambda_{s}\right) d x \approx \frac{1}{\left(1+\frac{\lambda_{b}^{0}}{3.5 \lambda_{s}}\right)^{3.5}},
$$

where $(b)$ is obtained by an approximated value of $\lambda_{b}^{0}$. Then we provide the density for idle LISs as $\lambda_{s}^{1}=p_{v 2} \lambda_{s}$, and $\lambda_{s}^{0}=\left(1-p_{v 2}\right) \lambda_{s}$ is the density for assisted LISs which are linked to the BS-User pairs.

Corollary 2: Note that $\lambda_{b}^{2}=\lambda_{s}^{0}$ is the density of BS-LISUser link in the system. Meanwhile, we can provide BS-User link $\lambda_{b}^{3}$ gives

$$
\lambda_{b}^{3}=\lambda_{b}^{0}-\lambda_{b}^{2}=\left(1-p_{v 1}\right) \lambda_{b}-\left(1-p_{v 2}\right) \lambda_{s},
$$

which is the probability that BS-User pairs fail to connect to a LIS. Note that $\lambda_{b}^{3}$ will approach to 0 since $\lambda_{b} \ll \lambda_{s}$. 


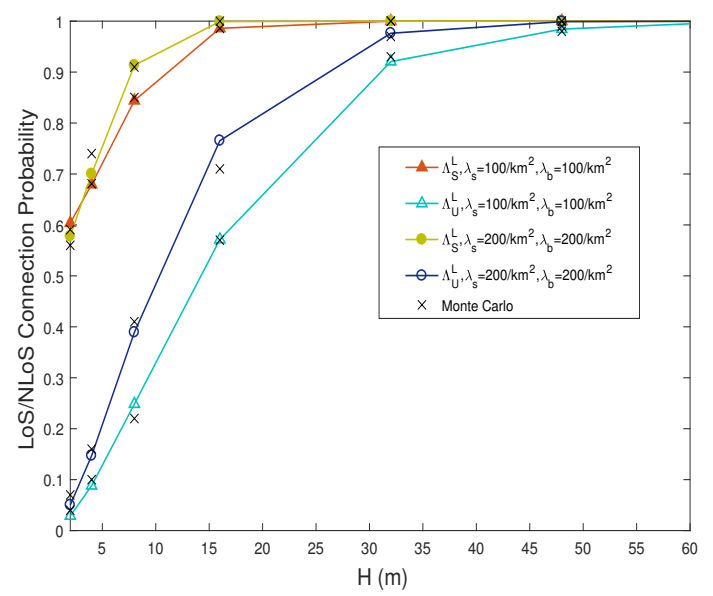

Fig. 3: . LoS connection probability for first hop in LIS and Second Hop LIS, where $\lambda_{u}=200 / \mathrm{m}^{2}, \alpha_{\mathrm{L}}=2, \alpha_{\mathrm{NL}}=3.4$.

\section{Reflection Path}

Next we analyze the reflected path from the serving BS to the assisting LIS for the typical user, and the reflective distance $z_{o}$ depends on two steps of user association.

Lemma 4: The probability that a user is associated with an LoS LIS in the reflect path is

$$
\begin{aligned}
\Lambda_{\mathrm{U}}^{(\mathrm{L})} & =\Lambda_{\mathrm{S}}^{(\mathrm{L})} \int_{0}^{2 \pi} \int_{H}^{\infty} \int_{0}^{\infty} \frac{1}{2 \pi} f_{\mathrm{B}}(x) f_{\mathrm{S}}^{(\mathrm{L})}\left(\sqrt{y_{o}^{2}-H^{2}}\right) \\
& \times p_{\mathrm{L}}\left(\sqrt{x_{o}^{2}+y_{o}^{2}-2 x_{o} y_{o} \cos \phi-H^{2}}\right) d x_{o} d y_{o} d \phi \\
& +\Lambda_{\mathrm{S}}^{(\mathrm{NL})} \int_{0}^{2 \pi} \int_{H}^{\infty} \int_{0}^{\infty} \frac{1}{2 \pi} f_{\mathrm{B}}(x) f_{\mathrm{S}}^{(\mathrm{NL})}\left(\sqrt{y_{o}^{2}-H^{2}}\right) \\
& \times p_{\mathrm{L}}\left(\sqrt{x_{o}^{2}+y_{o}^{2}-2 x_{o} y_{o} \cos \phi-H^{2}}\right) d x_{o} d y_{o} d \phi,
\end{aligned}
$$

where $\phi$ is the random included angle between $\angle \mathrm{S}_{o} \mathrm{~B}_{o} \mathrm{U}_{o}$, and $\phi \sim(0,2 \pi)$, and the probability that the reflected distance is NLoS link is $\Lambda_{\mathrm{U}}^{(\mathrm{NL})}=1-\Lambda_{\mathrm{U}}^{(\mathrm{L})}$.

Fig. 3 illustrates the LoS connection probability of the reflected path of user association from the typical user to the assisting LIS in (37) and LoS probability of the reflected path from the assisting LIS to the serving BS in (42), respectively. It is observers that both of them are increasing with a height of $H$. We note that the slope of the LoS probability in the reflection path is much higher than that in the first hop. The primary reason for this is that the typical user chooses the minimum reflection distance of $y_{o}$ for the maximize received signal by itself. However, the distance from serving $\mathrm{BS}$ to the assisting LIS follow the trigonometry as $z_{o}=\sqrt{x_{o}^{2}+y_{o}^{2}-2 x_{o} y_{o} \cos (\phi)}$. The results show the LoS probability for the reflection path converges to 1 faster when $\lambda_{s}=\lambda_{b}=200 / \mathrm{km}^{2}$ than when $\lambda_{s}=\lambda_{b}=100 / \mathrm{km}^{2}$. Note that the analytical results are computed numerically using (37) and (42), which are compared with Monte-Carlo simulations marked with ' $x$ ', the results are close and verify our analysis.

\section{Performance Analysis}

In this section, we provide general results for ASE and EE as performance indicators to analyze the capacity of LIS- assisted mmWave cellular networks.

\section{A. Area Spectrum Efficiency}

We use ASE to measure the network capacity, which is defined as the average throughput per unit spectrum and area [42]. We give the following definition for the ASE

$$
\mathcal{A}_{\mathrm{S}, o}=\lambda_{b}^{2} \mathcal{R}_{\mathrm{S}, o}+\lambda_{b}^{3} \mathcal{R}_{\mathrm{S}, d},
$$

where $\mathcal{R}_{\mathrm{S}, o}$ denotes the achievable rate of the BS-LIS-User link, and $o \in\{1,2\}$ indicates the MFs from a serving BS and typical users matched either directly with each other or via an assisting LIS. $\mathcal{R}_{\mathrm{S}, d}$ is the achievable rate without support from an LIS.

To evaluate the average achievable rate, we first derive the average rate $\mathcal{R}_{\mathrm{S}, o}$, which is given by the following theorem.

Theorem 1: The exact average achievable rate between the typical user and its intended BS in a BS-LIS-User link can be found as

$$
\mathcal{R}_{\mathrm{S}, o}=\Lambda_{\mathrm{S}}^{(\mathrm{L})} \mathcal{R}_{\mathrm{S}, o}^{(\mathrm{L})}+\Lambda_{\mathrm{S}}^{(\mathrm{NL})} \mathcal{R}_{\mathrm{S}, o}^{(\mathrm{NL})},
$$

where $\mathcal{R}_{\mathrm{S}, o}^{(v)}$ is the average achievable rate when a user is associated with an LoS or NLoS LIS, which is given by (45) at the top of the next page. $\Lambda_{\mathrm{S}}^{(v)}\left(y_{o}\right)$ is the LoS or NLoS connection probability given by (37) and (39), where $v$ denotes $\mathrm{L}$ or NL.

Proof 1: See Appendix A.

Theorem 2: The average achievable rate of the BS-User pairs without assistance from the LIS can be computed as

$$
\begin{aligned}
\mathcal{R}_{\mathrm{S}, d}=\frac{1}{\ln 2} \int_{0}^{\infty} \int_{0}^{\infty} \frac{1}{s} \mathrm{~A}_{d}\left(s, x_{o}\right) \\
\\
\times \mathrm{T}_{\mathrm{I}}\left(s, x_{o}\right) e^{-s \sigma^{2}} f_{\mathrm{B}}\left(x_{o}\right) d x_{o} d s
\end{aligned}
$$

where $\mathrm{T}_{I}\left(s, x_{o}\right)$ was given by (49), and we can obtain $\mathrm{A}_{d}\left(s, x_{o}\right)$ as

$$
\mathrm{A}_{d}\left(s, x_{o}\right)=1-\exp \left[-s P_{T} N_{b} N_{u} C x_{o}{ }^{-\alpha_{\mathrm{B}}}\right] .
$$

Corollary 3: In the direct BS-User link case, if a typical user is unable to be associated with any LIS, which denotes that the typical user is located in a low LIS interference area. Based on that, we neglect the LIS interference $\mathcal{I}_{S}$ to further simplify the expressions. The upper bound of the average achievable rate of the BS-User pairs can be expressed as follows

$$
\begin{aligned}
& \mathcal{R}_{\mathrm{S}, d}^{\mathrm{Upper}}=\frac{1}{\ln 2} \int_{0}^{\infty} \int_{0}^{\infty} \frac{1}{s}\left\{1-e^{-s N_{b} N_{u} C x_{o}^{-\alpha_{\mathrm{B}}}}\right\} \\
& \times e^{-s 2 \pi C n_{b} n_{u}\left(1-p_{v 1}\right) \lambda_{b} \frac{x_{o}^{2-\alpha_{\mathrm{B}}}}{\alpha_{\mathrm{B}}-2}} e^{-s \frac{\sigma^{2}}{P_{T}}} f_{\mathrm{B}}\left(x_{o}\right) d x_{o} d s \text {. }
\end{aligned}
$$

We derive the upper bound of ASE as follows

$$
\mathcal{A}_{\mathrm{S}, o}^{\mathrm{Upper}}=\lambda_{b}^{2} \mathcal{R}_{\mathrm{S}, o}+\lambda_{b}^{3} \mathcal{R}_{\mathrm{S}, d}^{\mathrm{Upper}} .
$$




$$
\begin{aligned}
& \mathcal{R}_{\mathrm{S}, o}^{(v)}=\frac{1}{2 \pi} \frac{1}{\ln 2} \\
& \times \int_{0}^{\infty} \frac{1}{s} \int_{0}^{2 \pi} \int_{H}^{\infty} \int_{0}^{\infty} \mathrm{A}_{\mathrm{S}, o}^{(v)}\left(s, x_{o}, y_{o}, \phi\right) \mathrm{T}_{I}\left(s, x_{o}\right) 2 \pi \lambda_{b} x_{o} e^{-\pi \lambda_{b} x_{o}{ }^{2}} f_{\mathrm{S}}^{(v)}\left(y_{o}\right) e^{-s \sigma^{2}} d x_{o} d y_{o} d \phi d s
\end{aligned}
$$

with

$$
\begin{aligned}
& \mathrm{A}_{\mathrm{S}, o}^{(v)}\left(s, x_{o}, y_{o}, \phi\right) \\
& =1-\exp \left[-s C P_{T}\left(\mathcal{N}_{(o)} x_{o}{ }^{-\alpha_{\mathrm{B}}}+M^{2} \widehat{\mathcal{N}}_{(o)} C y_{o}{ }^{-\alpha_{v}} \mathcal{Z}\left(x_{o}, y_{o}, \phi\right)+2 \sqrt{\frac{\widehat{\mathcal{N}}_{o} \mathcal{N}_{o}}{x_{o}^{\alpha_{\mathrm{B}}} y_{o} \alpha_{v}} \mathcal{Z}\left(x_{o}, y_{o}, \phi\right)}\right)\right]
\end{aligned}
$$

where

$$
\left\{\begin{array}{l}
\mathcal{N}_{(1)}=N_{b} N_{u}, \widehat{\mathcal{N}}_{(1)}=M^{2} \bar{n}_{b} \bar{n}_{u}, \text { when } o=1 \\
\mathcal{N}_{(2)}=\bar{n}_{b} \bar{n}_{u}, \widehat{\mathcal{N}}_{(2)}=M^{2} N_{b} N_{u}, \text { when } o=2
\end{array},\right.
$$

and

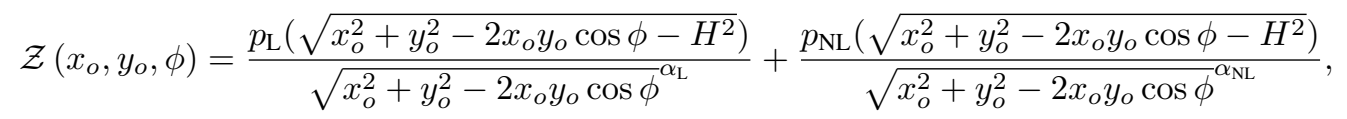

and

$$
\begin{aligned}
\mathrm{T}_{I}\left(s, x_{o}\right) & =\exp \left[-s 2 \pi C n_{u} n_{b}\left(1-p_{v 1}\right) \lambda_{b} P_{T}\left(\frac{x_{o}{ }^{2-\alpha_{\mathrm{B}}}}{\alpha_{\mathrm{B}}-2}\right.\right. \\
& \left.\left.\times 2 \pi\left(\lambda_{s} \chi_{\mathrm{o}}+p_{v 2} \lambda_{s}\left(\chi_{q}-\chi_{\mathrm{o}}\right)\right) C\left[\int_{H}^{\infty} u\left(\frac{p_{\mathrm{L}}\left(\sqrt{u^{2}-H^{2}}\right)}{u^{\alpha_{\mathrm{L}}}}+\frac{p_{\mathrm{NL}}\left(\sqrt{u^{2}-H^{2}}\right)}{u^{\alpha_{\mathrm{N}}}}\right) d u\right]^{2}\right)\right]
\end{aligned}
$$

\section{B. Energy Efficiency}

In this subsection, we evaluate the EE of the overall LISassisted system, which is defined as the ratio of the average throughput to the total average power consumption. Thus, the EE $\mathcal{E}_{\mathrm{S}, \mathrm{o}}$ for the LIS-assisted system under two different antenna schemes is given by

$$
\mathcal{E}_{\mathrm{S}, \mathrm{o}}=\frac{\lambda_{b}^{2} \mathcal{R}_{\mathrm{S}, \mathrm{o}}+\lambda_{b}^{3} \mathcal{R}_{\mathrm{S}, \mathrm{d}}}{P_{T} \lambda_{b}^{0}+M P_{s} \lambda_{s}^{0}}
$$

where $P_{s}$ denotes the power consumption of each adaptive phase shifter element from the active LIS density $\lambda_{s}^{0}[15,18]$, and $\mathcal{R}_{\mathrm{S}, \mathrm{o}}$ and $\mathcal{R}_{\mathrm{S}, \mathrm{d}}$ are the average achievable rate given by (44) and (50), respectively.

We also derive the upper bound of $\mathrm{EE} \mathcal{E}_{\mathrm{S}, \mathrm{o}}$ for the LISassisted system, which is given by

$$
\mathcal{E}_{\mathrm{S}, \mathrm{o}}^{\mathrm{Upper}}=\frac{\lambda_{b}^{2} \mathcal{R}_{\mathrm{S}, \mathrm{o}}+\lambda_{b}^{3} \mathcal{R}_{\mathrm{S}, \mathrm{d}}^{\mathrm{Upper}}}{P_{T} \lambda_{b}^{0}+M P_{s} \lambda_{s}^{0}}
$$

\section{Numerical Results AND Discussions}

In this section, we present the numerical results of the proposed LIS-assisted mmWave based cellular networks. Further, the performance analysis for average achievable rate, ASE and EE is highlighted. The system parameters are shown in Table I, unless otherwise specified.

The phase shift in real LIS systems is limited by the number of bits in its quantization. Fig. 4 compares ASE and EE with different bit quantization constraints $B$. We calculate the
TABLE I: Parameter Values.

\begin{tabular}{c|c}
\hline Parameters & Values \\
\hline \hline Number of antenna in BS $\left(N_{b}\right)$ & 2 \\
\hline Number of antenna in User $\left(N_{s}\right)$ & 2 \\
\hline Number of element in LIS $(M)$ & 64 \\
\hline Environment dependent constants $(a, b)$ & $9.6,0.28$ \\
\hline Transmit power of BS $\left(P_{T}\right)$ & $30 \mathrm{dBm}$ \\
\hline Power consumption of each element in LIS $\left(P_{s}\right)$ & $7 \mathrm{dBm}$ \\
\hline Density for BS $\lambda_{b}$ & $100,200 / \mathrm{km}^{2}$ \\
\hline Density for LIS $\lambda_{s}$ & $50 / \mathrm{km}^{2}$ \\
\hline Density for User $\lambda_{u}$ & $200 / \mathrm{km}^{2}$ \\
\hline Path loss exponent $f_{c}=73 \mathrm{GHz}[43]$ & $\alpha_{\mathrm{L}}=2, \alpha_{\mathrm{NL}}=3.4$ \\
\hline Available bandwidth $(\mathrm{BW})$ & $2 \mathrm{GHz}$ \\
\hline Height for LIS $(H)$ & $5 \mathrm{~m}$ \\
\hline Noise figure $(\mathrm{Nf})$ & $10 \mathrm{~dB}$ \\
\hline Noise Power $\left(\sigma_{o}^{2}\right)$ & $-170+\mathrm{Nf}$ \\
+10 log & \\
\hline
\end{tabular}

quantized phase shift $\varphi_{m}^{B} \in\left[0,2 \pi \frac{2^{B}-1}{2^{B}}\right)$ to replace the ideal phase shift $\varphi_{m}$ with its nearest realizable value. The error range is thus $\left|\varphi_{m}^{B}-\varphi_{m}\right|<\frac{0.5}{2^{B}}$. The results show that the margins of error for both ASE and EE between the ideal value and the quantized value are reduced by increasing the number of quantization bits: when $B$ is equal to 8 , the quantized values approach the optimal values.

Fig. 5(a) and Fig. 5(b) show the average achievable rate, ASE, and EE versus the number of elements of LIS $M$. From this Fig. 5(a), we can see that the average rates for both the direct and composite links increase with the $M$. Note that $\mathcal{R}_{\mathrm{S}, 2}$ is lower than $\mathcal{R}_{\mathrm{S}, 1}$ from (45) and $\mathcal{R}_{\mathrm{S}, \mathrm{d}}$ from (50) at the beginning. Therefore, the antenna scheme 1 may not 

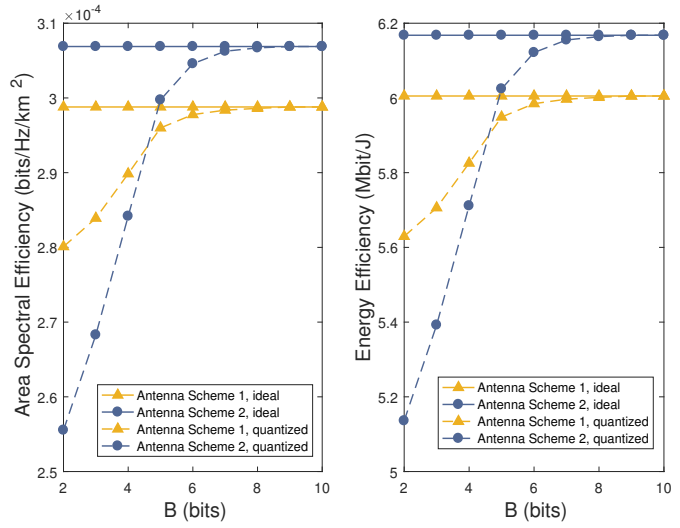

Fig. 4: Average achievable rate, ASE and EE versus number of quantization bits, where $M=16$.

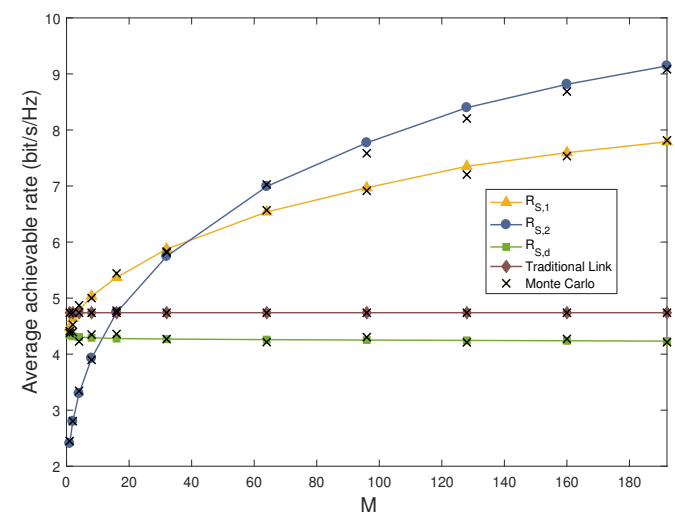

(a)
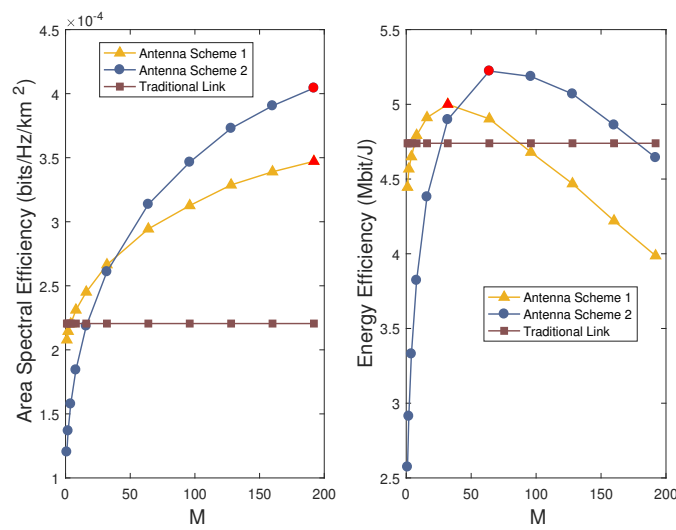

(b)

Fig. 5: Average achievable rate, ASE and EE versus number of LIS' element $M$, where $\lambda_{s}=100 / \mathrm{km}^{2}$.

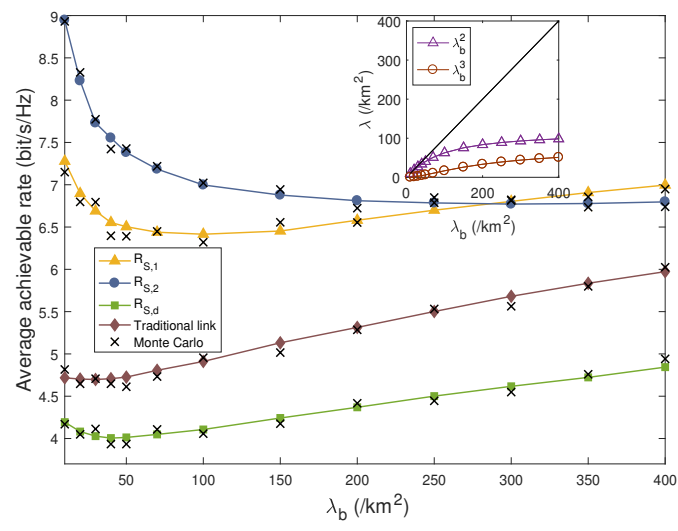

(a)
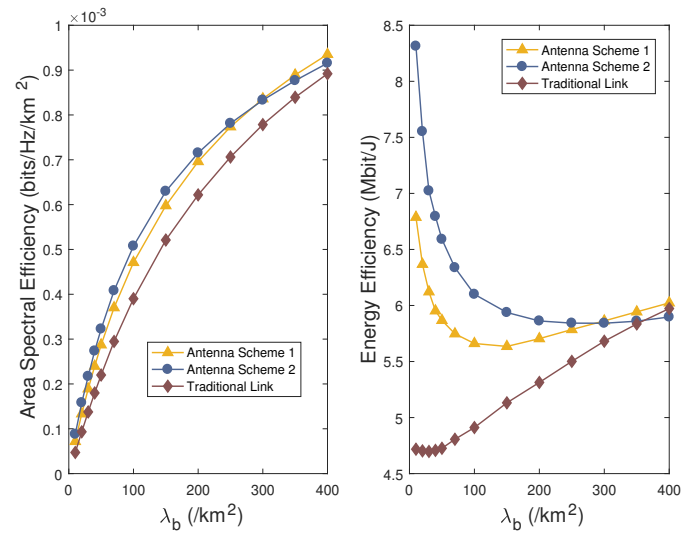

(b)

Fig. 6: Average achievable rate, ASE and EE versus density of BS $\lambda_{b}$, where $\lambda_{s}=200 / \mathrm{km}^{2}, M=32$.

effectively improve the ASE when the number of the elements of LIS $M$ increases to 32. More importantly, as the number of elements $M$ increases, $R_{\mathrm{S}, d}$ slowly rises even though there is an indirect relationship with $M$. The above is due to the main beam have being narrowed down with an increasing number of elements in the LISs in mmWave band; this will bring lower interference for ambient devices. The ASE in (43) and EE in (55) is shown in Fig. 5(b); both ASE and EE demonstrate an increasing trend for the average achievable rate, but EE decreases with extreme numbers of elements. If the number of elements in active LISs is over a specific value, the power consumption cannot be neglected, and it will significantly degrade the EE. For comparison, we introduce the average rate for traditional networks $\mathcal{R}_{\mathrm{BS}}$ without LIS. The traditional link only shows advantages when the LISs have a small number of elements. Note that the increase of the LIS' elements may bring the overload for EE.

Next, we plot the average achievable rate, ASE and EE versus the density of BS $\lambda_{b}$ in Fig. 6(a) and Fig. 6(b). We observe that the average achievable rates $\mathcal{R}_{\mathrm{S}, 1}, \mathcal{R}_{\mathrm{S}, 1}$, $\mathcal{R}_{\mathrm{S}, \mathrm{d}}$ are decreasing with density first, and then increasing, which demonstrates behavior opposite to traditional stochastic geometry networks. The reason is, at the beginning the interference from each BS increases due to the reflecting devices. 


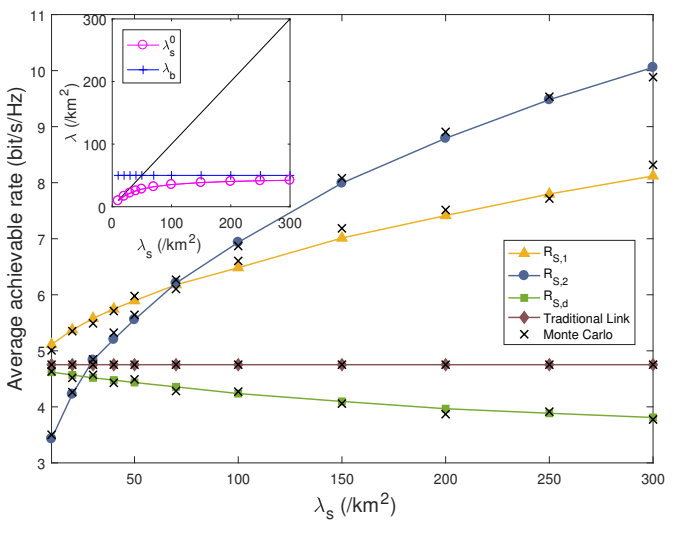

(a)
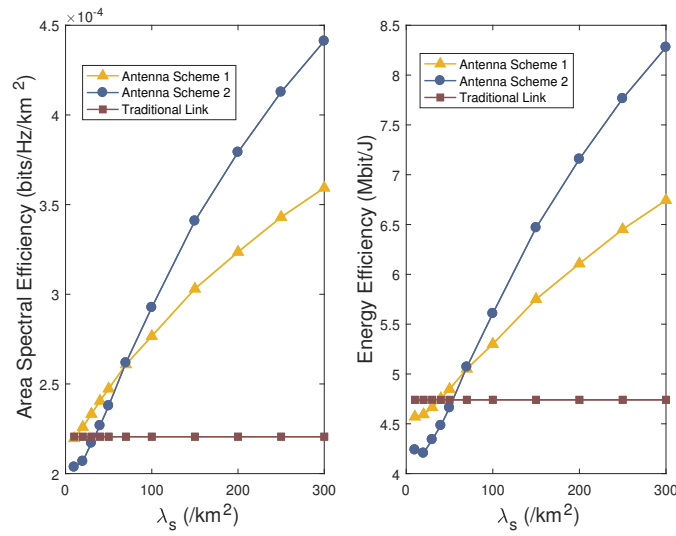

(b)

Fig. 7: Average achievable rate, ASE, EE versus density of LIS $\lambda_{s}$.

However, as the density of BSs increases, the distance between serving BS to assisting LIS and typical user decreases, and the number of active BSs who bring the ambient interference may limited by the constant requesting users; so the average data rate rises. In Fig. $6(\mathrm{~b})$, both $\mathcal{A}_{\mathrm{S}, 1}$ and $\mathcal{A}_{\mathrm{S}, 2}$ degrade at the beginning, since the transmit power consumption from BSs increases. Moreover, the energy efficiency in two antenna schemes $\mathcal{E}_{\mathrm{S}, 1}$ and $\mathcal{E}_{\mathrm{S}, 2}$ show a pattern similar to that of the average rate. Overall, for a given number of LISs, the LISassisted networks have a significant advantage in both EE and ASE over traditional networks, when $\lambda_{b}<\lambda_{s}$. However, as the BS density $\lambda_{b}$ continues to increase, it is possible that neither of the two antenna schemes in LIS-assisted networks will out perform traditional networks. The reason is the reflected interference and energy consumption in LIS-assisted networks, which demonstrates that LISs are not applicable in ultra-dense BSs networks.

Fig. 7(a) and Fig. 7(b) illustrate the average achievable rate, ASE and EE versus the density of LIS. The results indicate that the traditional average rate $\mathcal{R}_{\mathrm{BS}}$ is no longer invariant with LIS density $\lambda_{s}$. It can be observed that the average rate $\mathcal{R}_{\mathrm{S}, \mathrm{d}}$ of direct links declines with $\lambda_{s}$. As a result, LIS may not bring any benefit for traditional links and adding LIS devices will lead to high interference for all active nodes. It can be also

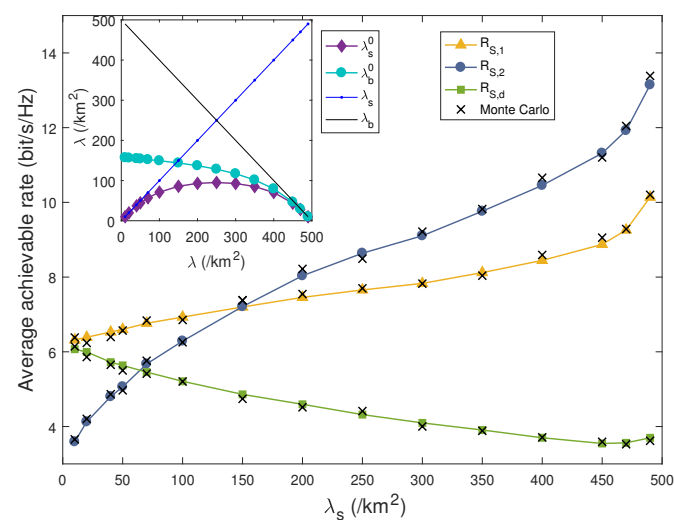

(a)
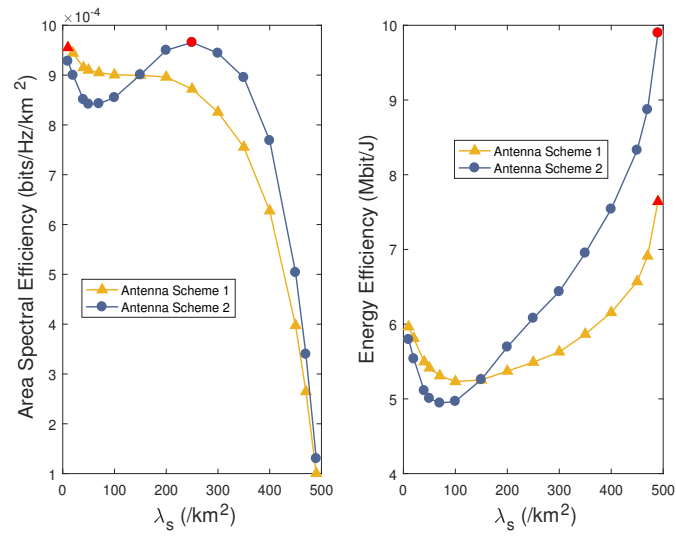

(b)

Fig. 8: Average achievable rate, ASE and EE versus density of LIS $\lambda_{s}$.

observed that antenna scheme 2 shows an obvious advantage when $\lambda_{s}>70 / \mathrm{km}^{2}$.

Subsequently, we investigate the trade-off between LIS density $\lambda_{s}$ and BS density $\lambda_{b}$, given a total sum density $\lambda_{s}+\lambda_{b}=500 / \mathrm{km}^{2}$. Fig. 8(a) shows that the average rate is optimised by increasing the density of LISs $\lambda_{s}$ and by decreasing the density of BSs $\lambda_{b}$. In Fig. 8(b), both antenna schemes show an optimal value of ASE when varying the BS density $\lambda_{b}$, which suggests that there exists an optimal LIS density factor to maximize the ASE. The reason is that at the beginning, as the density of LIS increases, both the density of assisting links and direct links goes up; but after an optimal point, the active BS-LIS-User links are limited by the number of BSs $\lambda_{b}^{0}$ (the sub-graph in Fig. 8(a)). However, we obtain the opposite result for EE, since LISs are dominating the network. The performance of each independent BS-User link is supported by ultra-dense LISs, the assisting LISs increase the performance with tiny energy consumption.

Fig. 9(a) and Fig. 9(b) show the average achievable rate, $\mathrm{ASE}$, and EE versus the height of LISs. Intuitively, as the height of the LISs rise, the performance in the direct link would drop because of the increasing LISs' interference. However, the LIS-supported rates $\mathcal{R}_{\mathrm{s}, 1}$ and $\mathcal{R}_{\mathrm{s}, 2}$ for antenna schemes 1 and 2 do not change monotonically; there is an 


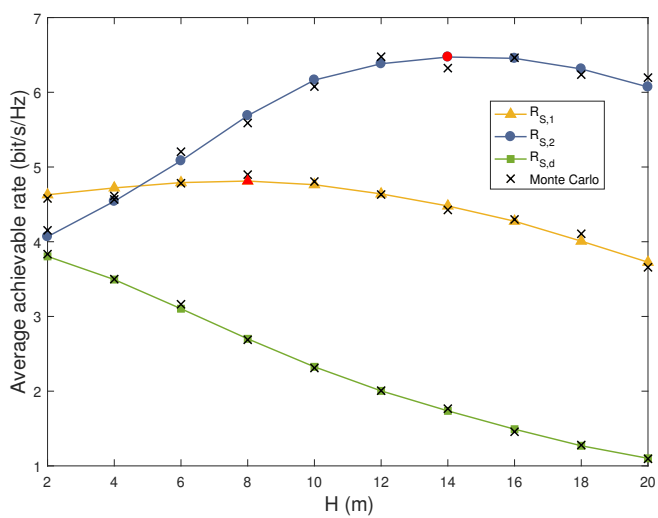

(a)
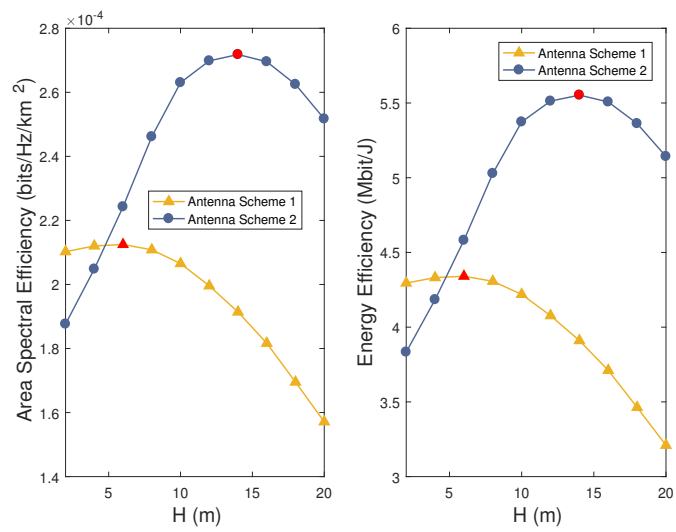

(b)

Fig. 9: Average achievable rate, ASE, EE versus the height of LIS, where $\lambda_{s}=200 / \mathrm{km}^{2}, M=12$.

optimal $H$ to maximize the average achievable rate since an increase in the height will raise the LoS probability for BS-LIS-User links. On the other hand, with further rising height, the path loss will significantly degrade the overall performance.

Fig. 10(a) and Fig. 10(b) provide the average achievable rate, ASE, and EE versus the number of antennas in BSs. The dashed curves obtained from (52) denote the upper-bound average rate in BS-User links, without assistance from an LIS. We observe that the upper bound curves can efficiently predict the performance behavior of $\mathcal{R}_{\mathrm{S}, d}$. It is shown that when the number of the antennas in BSs grows large, there is an obvious increase in the average achievable rate: the reason is that when the typical user is unable to be associated with its nearest LIS for support, the reflecting interference from ambient LISs will have less effect on BSs.

Furthermore, we can see that antenna scheme 1 is applicable to small LIS densities or low altitudes; in other words, antenna scheme 1 enhances weakly supported LIS networks. Once LIS gains advantages, such as broad LoS range and high density, antenna scheme 2 enormously reduces energy consumption and brings lower interference in the mmWave band.

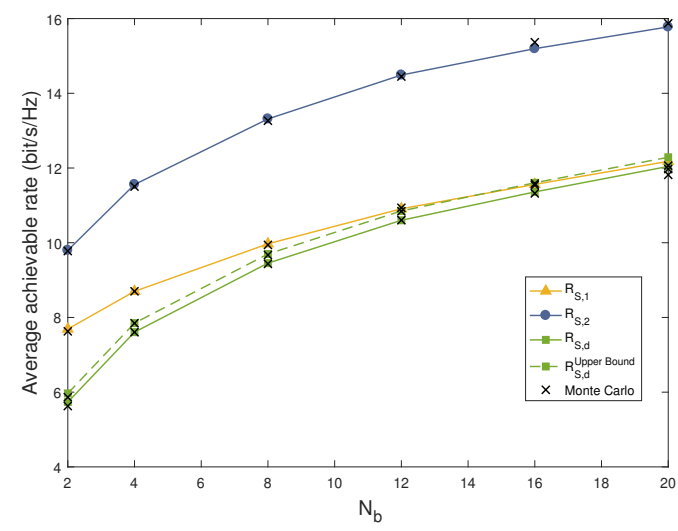

(a)
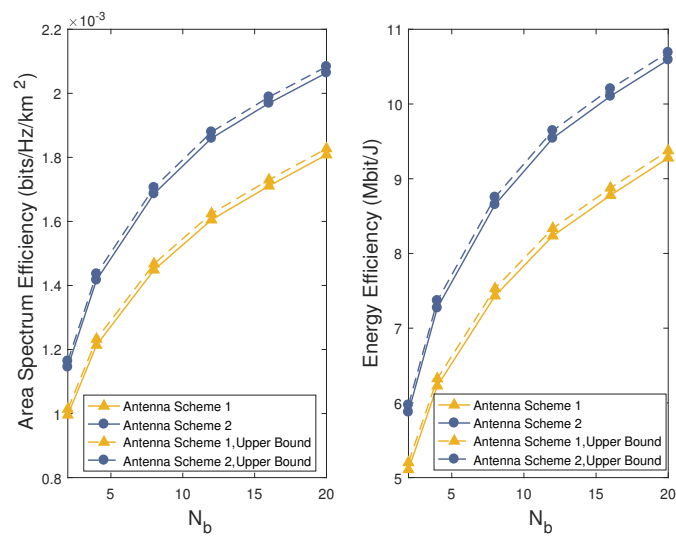

(b)

Fig. 10: Average achievable rate, ASE, EE versus the height of LIS, where $\lambda_{s}=100 / \mathrm{km}^{2}, \lambda_{b}=400 / \mathrm{km}^{2}, H=30 \mathrm{~m}$, $M=128$.

\section{CONClusions AND Future Work}

In this paper, we analyzed the performance of LIS-supported large-scale mmWave networks. We considered a composite two-step user association in system deployment constraints, which guarantees minimum path loss between each pair of nodes in a randomly located networks. Moreover, two types of antenna schemes of multiple ULA were employed to maximize the array gain in BS-LIS-User pairs. In addition, we examined the average achievable rate, ASE and EE, to analyze the LISassisted mmWave networks. Numerical results demonstrate that LIS-supported networks can achieve higher ASE and reduce energy consumption as compared to traditional networks, except in the case when BS density is greater than LIS density. Furthermore, there exists an optimal number of elements in LIS and density that maximizes EE and ASE. 


\section{Appendix A: Proof of Theorem 1}

Proof 2: The average achievable rate $\mathcal{R}_{\mathrm{S}, o}$ for BS-LIS-User links is calculated as

$$
\begin{aligned}
\mathcal{R}_{\mathrm{S}, o} & =\mathbb{E}\left[\log _{2}\left(1+\gamma_{\mathrm{S}}\right)\right] \\
& =\Lambda^{(\mathrm{LoS})} \mathbb{E}\left[\frac{1}{\ln 2} \int_{0}^{\infty} \frac{1}{s} \Upsilon_{o}^{(\mathrm{L})}(s) e^{-s} d s\right] \\
& +\Lambda^{(\mathrm{NLoS})} \mathbb{E}\left[\frac{1}{\ln 2} \int_{0}^{\infty} \frac{1}{s} \Upsilon_{o}^{(\mathrm{NL})}(s) e^{-s} d s\right],
\end{aligned}
$$

using [44, Lemma 1], we can compute $\Upsilon_{o}{ }^{(\mathrm{L})}(s)$ as

$$
\begin{aligned}
& \Upsilon_{o}{ }^{(\mathrm{L})}(s)=\frac{1}{2 \pi} \int_{0}^{2 \pi} \int_{H}^{\infty} \int_{0}^{\infty} \mathbb{E}\left[1-e^{-s \gamma_{\mathrm{s}}^{(\mathrm{L})}\left(s, x_{o}, y_{o}, \phi\right)}\right] \\
& \times f_{\mathrm{B}}\left(x_{o}\right) f_{\mathrm{S}}^{(\mathrm{L})}\left(y_{o}\right) d x_{o} d y_{o} d \phi \\
& \stackrel{(c)}{=} \frac{1}{2 \pi} \int_{0}^{2 \pi} \int_{H}^{\infty} \int_{0}^{\infty} \underbrace{\mathbb{E}\left[1-e^{-s \mathcal{Y}_{\mathrm{s}}^{(\mathrm{L})}\left(s, x_{o}, y_{o}, \phi\right)}\right]}_{\mathrm{A}_{\mathrm{S}}^{(\mathrm{L})}\left(s, x_{o}, y_{o}, \phi\right)} \\
& \times \underbrace{\mathbb{E}\left[e^{-s \mathcal{I}_{B}\left(s, x_{o}\right)}\right]}_{\mathrm{B}_{0}\left(s, x_{o}\right)} \underbrace{\mathbb{E}\left[e^{-s \mathcal{I}_{\mathrm{S}}(s)}\right]}_{\mathrm{C}_{\mathrm{S}}(s)} e^{-s \sigma^{2}} \\
& \times f_{\mathrm{B}}\left(x_{o}\right) f_{\mathrm{S}}^{(\mathrm{L})}\left(y_{o}\right) d x_{o} d y_{o} d \phi \text {, }
\end{aligned}
$$

where step $(c)$ is based on the fact that received composite signal and interference are independent in LIS-assisted networks, $A_{S, 1}^{(L)}$ is dependent on the LoS condition with second association probability $f_{\mathrm{S}}^{(\mathrm{L})}$. Under antenna scheme 1 this is

$$
\begin{aligned}
& \mathrm{A}_{\mathrm{S}, 1}^{(\mathrm{L})}\left(s, x_{o}, y_{o}, \theta\right)=\mathbb{E}\left[1-e^{-s \mathcal{Y}_{m}\left(s, x_{o}, y_{o}, \phi\right)}\right] \\
& =1-\mathbb{E}\left\{\exp \left[-s\left(P_{T}\left\|\mathbf{h}_{o}\right\|^{2} C x_{o}{ }^{-\alpha_{\mathrm{B}}}\right)\right]\right\} \\
& \times \mathbb{E}\left\{\exp \left[-s\left(P_{T}\left\|\mathbf{g}_{o} \boldsymbol{\Theta}_{\mathrm{o}} \mathbf{k}_{o}\right\|^{2} C^{2} y_{o}{ }^{-\alpha_{\mathrm{L}}} L\left(z_{o}\left(x_{o}, y_{o}, \phi\right)\right)\right)\right]\right\} \\
& =1-\exp \left[-s P_{T} C\left(\frac{N_{b} N_{u}}{x_{o}{ }^{\alpha_{\mathrm{B}}}}+\frac{M^{2} \bar{n}_{u} \bar{n}_{b}}{y_{o}^{\alpha_{\mathrm{L}}}} L\left(z_{o}\left(x_{o}, y_{o}, \phi\right)\right)\right)\right] \\
& \left.\times \exp \left[-2 s P_{T} M \sqrt{N_{b} N_{u} \bar{h}_{u} \bar{h}_{b}} C \frac{L\left(z_{o}\left(x_{o}, y_{o}, \phi\right)\right.}{\sqrt{x_{o}^{\alpha_{\mathrm{B}}} y_{o}^{\alpha_{\mathrm{L}}}}}\right)\right],
\end{aligned}
$$

and $\mathrm{A}_{\mathrm{S}, 2}^{(\mathrm{L})}$ denotes received composite signal under antenna scheme 2 , which can be expressed as

$$
\begin{aligned}
& \mathrm{A}_{\mathrm{S}, 2}^{(\mathrm{L})}\left(s, x_{o}, y_{o}, \phi\right)=1-\exp \left[-s P_{T} \bar{h}_{u} \bar{h}_{b} C x_{o}{ }^{-\alpha_{\mathrm{B}}}\right] \\
& \quad \times \exp \left[-s P_{T} M^{2} N_{b} N_{u} C y_{o}^{-\alpha_{\mathrm{L}}} L\left(z_{o}\left(x_{o}, y_{o}, \phi\right)\right)\right] \\
& \left.\quad \times \exp \left[-2 s P_{T} M \sqrt{N_{b} N_{u} \bar{h}_{u} \bar{h}_{b}} C \frac{L\left(z_{o}\left(x_{o}, y_{o}, \phi\right)\right.}{\sqrt{x_{o}^{\alpha_{\mathrm{B}}} y_{o}^{\alpha_{\mathrm{L}}}}}\right)\right],
\end{aligned}
$$

with

$$
L\left(z_{o}\right)=C\left(z_{o}{ }^{-\alpha_{\mathrm{L}}} p_{\mathrm{L}}\left(z_{o}\right)+z_{o}{ }^{-\alpha_{\mathrm{N}}} p_{\mathrm{NL}}\left(z_{o}\right)\right),
$$

and $z_{o}\left(x_{o}, y_{o}, \phi\right)=\sqrt{x_{o}^{2}+y_{o}^{2}-2 x_{o} y_{o} \cos \phi}$ denotes the distance from an assisting LIS to the associated BS, and $\mathrm{B}_{o}\left(s, x_{o}\right)$ in the interference from all active $\mathrm{BSs}$ set $\Phi_{b}^{0}$ with density $\lambda_{b}^{0}$, by applying the Laplace functional of the PPP, which is given by

$$
\begin{aligned}
\mathrm{B}_{o}\left(s, x_{o}\right) & =\mathbb{E}\left[e^{-s \mathcal{I}_{B}\left(x_{o}\right)}\right] \\
& =\exp \left[-2 \pi \lambda_{b}^{0} \int_{x_{o}}^{\infty}\left(1-e^{-s P_{T} \mathcal{N}_{i} C r^{-\alpha} \mathrm{B}}\right) r d r\right] .
\end{aligned}
$$

The interference from LISs which reflect interference $\mathrm{C}_{\mathrm{S}}(s)$ from other active BSs can be computed through

$$
\begin{aligned}
\mathrm{C}_{\mathrm{S}}(s) & =\mathbb{E}\left[e^{-s \mathcal{I}_{\mathrm{S}}(s)}\right] \\
& \approx \exp [-s \sum_{k \in \Phi_{s}^{1}} \underbrace{\mathbb{E}\left[\sum_{j \in \Phi_{b}^{1}} P_{T} \bar{h}_{b} L\left(y_{j}\right)\right]}_{\Omega} \chi_{i} \bar{h}_{u} L\left(z_{k}\right)] \\
& \times \exp [-s \sum_{k \in \Phi_{s}^{0}} \underbrace{\mathbb{E}\left[\sum_{j \in \Phi_{b}^{1}} P_{T} \bar{h}_{b} L\left(y_{j}\right)\right]}_{\Omega} \chi_{q} \bar{h}_{u} L\left(z_{k}\right)],
\end{aligned}
$$

$\Omega$ is the integration interference from all active BSs for one typical LIS, which from Campbell's theorem [45] is given as

$$
\begin{aligned}
\Omega & \approx 2 \pi P_{T} \lambda_{s}^{1} \chi_{\mathrm{o}} \bar{h}_{b} C \\
& \times \int_{H}^{\infty} u\left(\frac{p_{\mathrm{L}}\left(\sqrt{u^{2}-H^{2}}\right)}{u^{\alpha_{\mathrm{L}}}}+\frac{p_{\mathrm{NL}}\left(\sqrt{u^{2}-H^{2}}\right)}{u^{\alpha_{\mathrm{N}}}}\right) d u .
\end{aligned}
$$

Then we can expand the $\mathrm{C}_{\mathrm{S}}(s)$ again as

$$
\begin{aligned}
& \mathrm{C}_{\mathrm{S}}(s)= \\
& \exp \left\{-s 2 \pi \lambda_{s}^{1} \int_{H}^{\infty} p_{\mathrm{L}}\left(\sqrt{r^{2}-H^{2}}\right)\left(1-e^{-\frac{s \Omega \chi_{i} \bar{h}_{u} C}{r^{\alpha} \mathrm{L}}}\right) r d r\right. \\
& \left.\quad+\int_{H}^{\infty} p_{\mathrm{N}}\left(\sqrt{r^{2}-H^{2}}\right)\left(1-e^{-\frac{s \Omega \chi_{i} \bar{h}_{u} C}{r^{\alpha} \mathrm{N}}}\right) r d r\right\} \\
& \quad \times \exp \left\{-s 2 \pi \lambda_{s}^{0} \int_{H}^{\infty} p_{\mathrm{L}}\left(\sqrt{r^{2}-H^{2}}\right)\left(1-e^{-\frac{s \Omega \chi_{q} \bar{h}_{u} C}{r^{\alpha} \mathrm{L}}}\right) r d r\right. \\
& \left.\quad+\int_{H}^{\infty} p_{\mathrm{N}}\left(\sqrt{r^{2}-H^{2}}\right)\left(1-e^{-\frac{s \Omega \chi_{q} \bar{h}_{u} C}{r^{\alpha}}}\right) r d r\right\} \cdot \text { (A.9) }
\end{aligned}
$$

Likewise, we can derive $\Upsilon_{o}{ }^{(\mathrm{NL})}(s)$ in the NLoS link as

$$
\begin{gathered}
\Upsilon_{o}{ }^{(\mathrm{NL})}(s)=\frac{1}{2 \pi} \int_{0}^{2 \pi} \int_{0}^{\infty} \int_{0}^{\infty} \underbrace{\mathbb{E}\left[1-e^{-s \mathcal{Y}_{m}\left(s, x_{o}, y_{o}, \phi\right)}\right]}_{\mathrm{A}_{\mathrm{S}, o}^{(\mathrm{NL})}\left(s, x_{o}, y_{o}, \phi\right)} \\
\times \mathrm{B}_{\mathrm{o}}\left(s, x_{o}\right) \mathrm{C}_{S}\left(s, y_{o}\right) f_{B}\left(x_{o}\right) f_{\mathrm{S}}^{(\mathrm{NL})}\left(y_{o}\right) d x_{o} d y_{o} d \phi .
\end{gathered}
$$

Using a similar approach in (A.3), $\mathrm{A}_{\mathrm{S}, 1}^{(\mathrm{NL})}\left(s, x_{o}, y_{o}, \theta\right)$ is derived as

$$
\begin{aligned}
\mathrm{A}_{\mathrm{S}, 1}^{(\mathrm{NL})} & \left(s, x_{o}, y_{o}, \theta\right)=1-\exp \left[-s P_{T} N_{b} N_{u} C x_{o}{ }^{-\alpha_{\mathrm{B}}}\right] \\
& \times \exp \left[-s P_{T} M^{2} \bar{h}_{b} \bar{h}_{u} C y_{o}{ }^{-\alpha_{\mathrm{NL}}} L\left(z_{o}\left(x_{o}, y_{o}, \phi\right)\right)\right],
\end{aligned}
$$


applying the antenna scheme 2 as in (A.4), we can obtain $\mathrm{A}_{\mathrm{S}, 2}^{(\mathrm{NL})}\left(s, x_{o}, y_{o}, \theta\right)$. Substituting (A.3), (A.4) (A.6) and (A.9) into (A.2), we obtain (A.1), which completes the proof.

\section{REFERENCES}

[1] Cisco, "Cisco visual networking index: Global mobile data traffic forecast update: 2016-2021 white paper," Feb. 2017.

[2] T. J. Cui, M. Q. Qi, X. Wan, J. Zhao, and Q. Cheng, "Coding metamaterials, digital metamaterials and programmable metamaterials," Light: Science \& Applications, vol. 3, no. 10, p. e218, Oct. 2014.

[3] W. Tang, J. Y. Dai, M. Z. Chen, K.-K. Wong, X. Li, X. Zhao, S. Jin, Q. Cheng, and T. J. Cui, "Mimo transmission through reconfigurable intelligent surface: System design, analysis, and implementation," arXiv preprint arXiv:1912.09955, 2019.

[4] W. Tang, M. Z. Chen, X. Chen, J. Y. Dai, Y. Han, M. Di Renzo, Y. Zeng, S. Jin, Q. Cheng, and T. J. Cui, "Wireless communications with reconfigurable intelligent surface: Path loss modeling and experimental measurement," arXiv preprint arXiv:1911.05326, 2019.

[5] A. Forouzmand, M. M. Salary, S. Inampudi, and H. Mosallaei, "A tunable multigate indium-tin-oxide-assisted all-dielectric metasurface," Advanced Optical Materials, vol. 6, no. 7, p. 1701275, 2018.

[6] T. J. Cui, S. Liu, and L. Zhang, "Information metamaterials and metasurfaces," Journal of Materials Chemistry C, vol. 5, no. 15, pp. 3644-3668, Mar. 2017.

[7] L. Zhang, X. Q. Chen, S. Liu, Q. Zhang, J. Zhao, J. Y. Dai, G. D. Bai, X. Wan, Q. Cheng, G. Castaldi et al., "Space-time-coding digital metasurfaces," Nature communications, vol. 9, no. 1, p. 4334, Oct. 2018.

[8] M.-A. Badiu and J. P. Coon, "Communication through a large reflecting surface with phase errors," arXiv preprint arXiv:1906.10751, 2019.

[9] Y. Han, W. Tang, S. Jin, C. Wen, and X. Ma, "Large intelligent surfaceassisted wireless communication exploiting statistical csi," IEEE Trans. Veh. Technol., vol. 68, no. 8, pp. 8238-8242, 2019.

[10] Q. Wu and R. Zhang, "Intelligent reflecting surface enhanced wireless network: Joint active and passive beamforming design," in 2018 IEEE Global Communications Conference (GLOBECOM), Dec 2018, pp. 1-6.

[11] S. Hu, F. Rusek, and O. Edfors, "Beyond Massive MIMO: The potential of data transmission with large intelligent surfaces," IEEE Trans. Signal Process., vol. 66, no. 10, pp. 2746-2758, May 2018.

[12] E. Basar, "Large intelligent surface-based index modulation: A new beyond MIMO paradigm for 6G," arXiv preprint arXiv:1904.06704, 2019.

[13] M. Jung, W. Saad, Y. Jang, G. Kong, and S. Choi, "Uplink data rate in large intelligent surfaces: Asymptotic analysis under channel estimation errors," in submitted to Proc. of IEEE Wireless Communications and Networking Conference (WCNC), Marrakech, Morocco, 2019.

[14] T. Hou, Y. Liu, Z. Song, X. Sun, Y. Chen, L. Hanzo et al., "Reconfigurable intelligent surface aided noma networks," arXiv preprint arXiv:1912.10044, 2019.

[15] C. Huang, A. Zappone, G. C. Alexandropoulos, M. Debbah, and C. Yuen, "Reconfigurable intelligent surfaces for energy efficiency in wireless communication," IEEE Trans. Wireless Commun., vol. 18, no. 8 , pp. 4157-4170, Aug. 2019.

[16] C. Zhong, H. A. Suraweera, G. Zheng, I. Krikidis, and Z. Zhang, "Wireless information and power transfer with full duplex relaying," IEEE Trans. Commun., vol. 62, no. 10, pp. 3447-3461, Oct 2014.

[17] N. Deng, S. Zhang, W. Zhou, and J. Zhu, "A stochastic geometry approach to energy efficiency in relay-assisted cellular networks," in 2012 IEEE Global Communications Conference (GLOBECOM), Dec 2012, pp. 3484-3489.

[18] E. Björnson, Ö. Özdogan, and E. G. Larsson, "Intelligent reflecting surface vs. decode-and-forward: How large surfaces are needed to beat relaying?" arXiv preprint arXiv:1906.03949, 2019.

[19] K. Ntontin, J. Song, and M. Di Renzo, "Multi-antenna relaying and reconfigurable intelligent surfaces: End-to-end snr and achievable rate," arXiv preprint arXiv:1908.07967, 2019.

[20] X. Yu, D. Xu, and R. Schober, "Enabling secure wireless communications via intelligent reflecting surfaces," arXiv preprint arXiv:1904.09573, 2019.

[21] X. Guan, Q. Wu, and R. Zhang, "Intelligent reflecting surface assisted secrecy communication via joint beamforming and jamming," arXiv preprint arXiv:1907.12839, 2019.

[22] J. Chen, Y.-C. Liang, Y. Pei, and H. Guo, "Intelligent reflecting surface: A programmable wireless environment for physical layer security," arXiv preprint arXiv:1905.03689, 2019.
[23] M. Cui, G. Zhang, and R. Zhang, "Secure wireless communication via intelligent reflecting surface," IEEE Wireless Commun. Lett., pp. 1-1, May 2019.

[24] M. R. Akdeniz, Y. Liu, M. K. Samimi, S. Sun, S. Rangan, T. S. Rappaport, and E. Erkip, "Millimeter Wave channel modeling and cellular capacity evaluation," IEEE J. Sel. Areas Commun., vol. 32, no. 6 , pp. 1164-1179, June 2014.

[25] T. Bai and R. W. Heath, "Coverage and rate analysis for millimeter-wave cellular networks," IEEE Trans. Wireless Commun., vol. 14, no. 2, pp. 1100-1114, Feb 2015.

[26] K. Ntontin, M. Di Renzo, J. Song, F. Lazarakis, J. de Rosny, D.-T. Phan-Huy, O. Simeone, R. Zhang, M. Debbah, G. Lerosey et al., "Reconfigurable intelligent surfaces vs. relaying: Differences, similarities, and performance comparison," arXiv preprint arXiv:1908.08747, 2019.

[27] X. Tan, Z. Sun, D. Koutsonikolas, and J. M. Jornet, "Enabling indoor mobile millimeter-wave networks based on smart reflect-arrays," in IEEE INFOCOM 2018 - IEEE Conference on Computer Communications, April 2018, pp. 270-278.

[28] M. Haenggi, J. G. Andrews, F. Baccelli, O. Dousse, and M. Franceschetti, "Stochastic geometry and random graphs for the analysis and design of wireless networks," IEEE Journal on Selected Areas in Communications, vol. 27, no. 7, pp. 1029-1046, Sep. 2009.

[29] J. G. Andrews, F. Baccelli, and R. K. Ganti, "A tractable approach to coverage and rate in cellular networks," IEEE Trans. Commun., vol. 59, no. 11, pp. 3122-3134, November 2011.

[30] Z. Chen and E. Björnson, "Channel hardening and favorable propagation in Cell-free massive MIMO with stochastic geometry," IEEE Trans. Commun., vol. 66, no. 11, pp. 5205-5219, Nov 2018.

[31] Y. Zhu, L. Wang, K. Wong, and R. W. Heath, "Secure communications in millimeter wave ad hoc networks," IEEE Trans. Wireless Commun., vol. 16, no. 5, pp. 3205-3217, May 2017.

[32] A. Thornburg, T. Bai, and R. W. Heath, "Performance analysis of outdoor mmwave ad hoc networks," IEEE Trans. Signal Process., vol. 64, no. 15, pp. 4065-4079, Aug. 2016.

[33] Y. Niu, Y. Li, D. Jin, L. Su, and A. V. Vasilakos, "A survey of millimeter wave communications (mmWave) for 5G: opportunities and challenges," Wireless networks, vol. 21, no. 8, pp. 2657-2676, Nov. 2015.

[34] Q. Wu and R. Zhang, "Weighted sum power maximization for intelligent reflecting surface aided swipt," IEEE Wireless Commun. Lett., pp. 1-1, 2019.

[35] E. Turgut and M. C. Gursoy, "Downlink analysis in unmanned aerial vehicle (uav) assisted cellular networks with clustered users," IEEE Access, vol. 6, pp. 36313-36324, 2018.

[36] S. Sun, T. S. Rappaport, R. W. Heath, A. Nix, and S. Rangan, "MIMO for millimeter-wave wireless communications: beamforming, spatial multiplexing, or both?" IEEE Commun. Mag., vol. 52, no. 12, pp. 110121, December 2014.

[37] Z. Xiao, T. He, P. Xia, and X. Xia, "Hierarchical codebook design for beamforming training in millimeter-wave communication," IEEE Trans. Wireless Commun., vol. 15, no. 5, pp. 3380-3392, May 2016.

[38] T. S. Rappaport, F. Gutierrez, E. Ben-Dor, J. N. Murdock, Y. Qiao, and J. I. Tamir, "Broadband millimeter-wave propagation measurements and models using adaptive-beam antennas for outdoor urban cellular communications," IEEE Trans. Antennas Propag., vol. 61, no. 4, pp. 1850-1859, April 2013.

[39] H. Jo, Y. J. Sang, P. Xia, and J. G. Andrews, "Heterogeneous cellular networks with flexible cell association: A comprehensive downlink sinr analysis," IEEE Trans. Wireless Commun., vol. 11, no. 10, pp. 34843495, October 2012.

[40] S. Lee and K. Huang, "Coverage and economy of cellular networks with many base stations," IEEE Commun. Lett., vol. 16, no. 7, pp. 1038-1040, July 2012.

[41] A. Al-Hourani, S. Kandeepan, and S. Lardner, "Optimal lap altitude for maximum coverage," IEEE Commun. Lett., vol. 3, no. 6, pp. 569-572, Dec 2014.

[42] M. . Alouini and A. J. Goldsmith, "Area spectral efficiency of cellular mobile radio systems," IEEE Trans. Veh. Technol., vol. 48, no. 4, pp. 1047-1066, July 1999.

[43] S. Deng, M. K. Samimi, and T. S. Rappaport, " $28 \mathrm{GHz}$ and 73 $\mathrm{GHz}$ millimeter-wave indoor propagation measurements and path loss models," in 2015 IEEE International Conference on Communication Workshop (ICCW), June 2015, pp. 1244-1250.

[44] K. A. Hamdi, "Capacity of mrc on correlated rician fading channels," IEEE Trans. Commun., vol. 56, no. 5, pp. 708-711, May 2008. 
[45] F. Baccelli, B. Błaszczyszyn et al., "Stochastic geometry and wireless networks: Volume ii applications," Foundations and Trends $₫$ in Networking, vol. 4, no. 1-2, pp. 1-312, 2010.

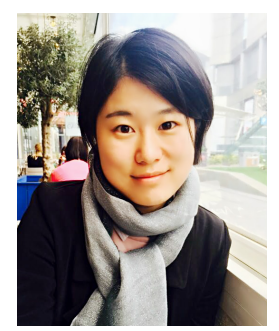

Yongxu Zhu (S'16-M'19) received the Ph.D degree in Electrical Engineering from University College London in 2017. From 2017 to 2019, she was a Research Associate with Loughborough University. She is currently a Lecturer (Assistant Professor) with the Division of Computer Science and informatics, London South Bank University, since 2019. She has served as an Editor of the IEEE Wireless Communication Letters. Her research interests include large intelligent surface Communications, UAV communications, wireless edge caching, millimeter-wave communications, heterogeneous cellular networks, physical-layer security and Blockchain.

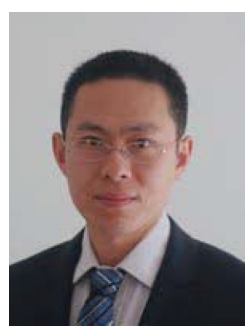

Gan Zheng (S'05-M'09-SM'12) received the BEng and the MEng from Tianjin University, Tianjin, China, in 2002 and 2004, respectively, both in Electronic and Information Engineering, and the $\mathrm{PhD}$ degree in Electrical and Electronic Engineering from The University of Hong Kong in 2008. He is currently Reader of Signal Processing for Wireless Communications in the Wolfson School of Mechanical, Electrical and Manufacturing Engineering, Loughborough University, UK. His research interests include machine learning for communications, UAV communications, mobile edge caching, full-duplex radio, and wireless power transfer. He is the first recipient for the 2013 IEEE Signal Processing Letters Best Paper Award, and he also received 2015 GLOBECOM Best Paper Award, and 2018 IEEE Technical Committee on Green Communications \& Computing Best Paper Award. He was listed as a Highly Cited Researcher by Thomson Reuters/Clarivate Analytics in 2019. He currently serves as an Associate Editor for IEEE Communications Letters and IEEE Wireless Communications Letters.

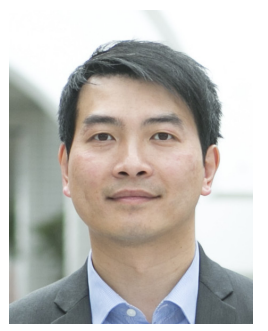

Kai-Kit Wong (M'01-SM'08-F'16) received the $\mathrm{BEng}$, the MPhil, and the PhD degrees, all in Electrical and Electronic Engineering, from the Hong Kong University of Science and Technology, Hong Kong, in 1996, 1998, and 2001, respectively. After graduation, he took up academic and research positions at the University of Hong Kong, Lucent Technologies, Bell-Labs, Holmdel, the Smart Antennas Research Group of Stanford University, and the University of Hull, UK. He is Chair in Wireless Communications at the Department of Electronic and Electrical Engineering, University College London, UK.

His current research centers around $5 \mathrm{G}$ and beyond mobile communications. $\mathrm{He}$ is a co-recipient of the 2013 IEEE Signal Processing Letters Best Paper Award and the 2000 IEEE VTS Japan Chapter Award at the IEEE Vehicular Technology Conference in Japan in 2000, and a few other international best paper awards. He is Fellow of IEEE and IET and is also on the editorial board of several international journals. He is the Editor-in-Chief for IEEE Wireless Communications Letters since 2020. 